\title{
Highlighting Exact Matching via Marking Strategies for Ad hoc Document Ranking with Pretrained Contextualized Language Models
}

\section{Lila BOUALILI ( $\sim$ lila.boualili@irit.fr)}

IRIT: Institut de recherche en informatique de Toulouse https://orcid.org/0000-0002-9985-131X Jose Moreno

IRIT: Institut de recherche en informatique de Toulouse

Mohand Boughanem

IRIT: Institut de recherche en informatique de Toulouse

\section{Research Article}

Keywords: Deep Learning, Pretrained Language Models, Ad hoc Ranking, Exact Term-Matching

Posted Date: February 21st, 2022

DOI: https://doi.org/10.21203/rs.3.rs-550456/v1

License: (c) (i) This work is licensed under a Creative Commons Attribution 4.0 International License.

Read Full License 


\title{
Highlighting Exact Matching via Marking Strategies for Ad hoc Document Ranking with Pretrained Contextualized Language Models
}

\author{
Lila Boualili · Jose G. Moreno • Mohand \\ Boughanem
}

Received: date / Accepted: date

\begin{abstract}
Pretrained language models (PLMs) exemplified by BERT have proven to be remarkably effective for ad hoc ranking. As opposed to preBERT models that required specialized neural components to capture different aspects of query-document relevance, PLMs are solely based on transformers where attention is the only mechanism used for extracting signals from term interactions. Thanks to the transformer's cross-match attention, BERT was found to be an effective soft matching model. However, exact matching is still an essential signal for assessing the relevance of a document to an informationseeking query aside from semantic matching. We assume that BERT might benefit from explicit exact match cues to better adapt to the relevance classification task. In this work, we explore strategies for integrating exact matching signals using marker tokens to highlight exact term-matches between the query and the document. We find that this simple marking approach significantly improves over the common vanilla baseline. We empirically demonstrate the effectiveness of our approach through exhaustive experiments on two standard ad hoc benchmarks. Results show that explicit exact match cues conveyed by marker tokens are beneficial for BERT and ELECTRA variant to achieve higher or at least comparable performance. Our findings support that traditional information retrieval cues such as exact matching are still valuable for large pretrained contextualized models such as BERT.
\end{abstract}

L. Boualili

IRIT, University of Toulouse 3, Toulouse, France

E-mail: lila.boualili@irit.fr

J.G. Moreno

IRIT, University of Toulouse 3, Toulouse, France

E-mail: jose.moreno@irit.fr

M. Boughanem

IRIT, University of Toulouse 3, Toulouse, France

E-mail: mohand.boughanem@irit.fr 
Table 1: Extracts from top ranked passages by Vanilla BERT for the query: "causes of left ventricular hypertrophy".

\begin{tabular}{ll}
\hline ID & Passage \\
\hline 47203 & $\begin{array}{l}\text { Causes of Right Ventricular Hypertrophy. There are four usual } \\
\text { causes of right ventricular hypertrophy... }\end{array}$ \\
5197133 & $\begin{array}{l}\text { The last common cause of right ventricular hypertrophy is the } \\
\text { ventricular septal defect... }\end{array}$ \\
7504775 & $\begin{array}{l}\text { The most common causes of right ventricle hypertrophy (RVH) } \\
\text { are diseases that damage the lung... }\end{array}$ \\
\hline
\end{tabular}

Keywords Deep Learning · Pretrained Language Models · Ad hoc Ranking · Exact Term-Matching

\section{Introduction}

Pretrained Language Models (PLMs), such as BERT (Devlin et al., 2019), ELECTRA (Clark et al. 2020) and T5 (Raffel et al., 2020), have become the core components for building highly effective ranking models. The success of PLMs is largely owed to the heavy pre-training on language modeling objectives on the one hand, and learning deeply-contextualized representations of input sequences using the transformer architecture (Vaswani et al., 2017) on the other. Thanks to the fine-tuning strategy and the availability of large publicly-released training datasets, applying a PLM to document ranking is straightforward. Nogueira and Cho (2019) was the first to propose a simple application of BERT to text ranking using fine-tuning on the large public MS MARCO (Nguyen et al. 2016) dataset. In this work, BERT was deployed as a relevance classifier trained to estimate the probability each document is "relevant" w.r.t a given query.

Compared to the first wave of neural ranking models including DRMM (Guo et al., 2016), DUET(Mitra et al., 2017), and KNRM(Xiong et al., 2017), referred to as pre-BERT models, BERT and its variants do not appear to require any specialized neural architectural components to capture different aspects of relevance between a query and a document (Yates et al., 2021). The same architecture based on homogeneous transformer layers is employed regardless of the downstream task. Qiao et al. (2019) study the behaviour of BERT for ranking and revealed that it focuses more on document terms that directly match the query. Compared to pre-BERT models such as ConvKNRM (Dai et al. 2018) that prefer terms related to the query in search, BERT's pretraining on surrounding contexts favors text sequence pairs that are closer in their semantic meaning (Qiao et al., 2019). Qiao et al. (2019) conclude that BERT can be considered as an interaction-based sequence-tosequence soft matching model that owes its effectiveness to the transformer's cross-match attention. While soft semantic matching is, undeniably, a valuable 
Table 2: Extracts from top ranked passages by MarkedBERT for the query: "causes of left ventricular hypertrophy".

\begin{tabular}{ll}
\hline ID & Passage \\
\hline 8332546 & $\begin{array}{l}\text { Left ventricular hypertrophy can occur when...show evidence of } \\
\text { left ventricular hypertrophy at... }\end{array}$ \\
47203 & $\begin{array}{l}\text { Causes of Right Ventricular Hypertrophy. There are four usual } \\
\text { causes of right ventricular hypertrophy... } \\
\text { Left ventricular hypertrophy is a thickening of the wall of the } \\
\text { heart's main pumping chamber... }\end{array}$ \\
\hline
\end{tabular}

signal for relevance that alleviates the vocabulary mismatch problem, a ranking model needs proper handling of exact matching cues as well (Guo et al. 2016 . Luan et al., 2020). Let us take the following query: "Causes of left ventricular hypertrophy" form the MS MARCO passage ranking task, as an example. Table 1 reports extracts from the top passages retrieved by BERT. We can see that all top ranked passages are related to "right ventricular hypertrophy" due to the soft matching between "left" and "right". This example is a reminder of the importance of exact matching for relevance ranking. Boualili et al. (2020) suggest that a PLM like BERT can benefit from explicit exact matching signals for passage ranking. The authors propose MarkedBERT, a model that uses marker tokens to convey exact matches between the document and query terms from the input sequence. Exact term-matching integration via marking has proven to induce significant gains on the MSMARCO passage ranking task over "Vanilla" BERT (Nogueira and Cho, 2019). Analysis of the attention shows that marker tokens bring more focus on the exact matches allowing more relevant documents to be ranked higher. Table2 2 shows extracts from top ranked passages with MarkedBERT for the query "Causes of left ventricular hypertrophy" where we can count more documents related to "left ventricular hypertrophy" without explicit bias, since the passage 47203 ranked first by BERT is still ranked high (second) by MarkedBERT.

In this work, we follow the same hypothesis stating that exact matching cues can enhance PLMs and extend the previously proposed marking-based approach to ad hoc document ranking. We introduce new simple marking strategies and conduct extensive experiments to determine the contribution of exact match marking on PLMs like BERT and ELECTRA on standard ad hoc benchmarks. We empirically demonstrate the effectiveness of explicit exact match marking across different experimental scenarios. Since our approach aims at injecting an established traditional IR cue to recent pretrained transformers, we study the effectiveness of our models with interpolating the traditional BM25 scores. We find that best match scores obtained by BM25 are still valuable since they contribute to the end-to-end effectiveness. Furthermore, the marking-based models require less intervention from BM25 scores to achieve better ranking performance thant the vanilla baseline. 
Our main contributions can be summarized as follows:

- We introduce a new simple and unique marker token for highlighting all the exact term-matches without distinction and explore two marking strategies: document and pair marking.

- We conduct extensive experiments on standard TREC ad-hoc benchmark collections.

- We study the contribution of exact match scores from a bag-of-words model to the end-to-end effectiveness of our models.

- We compare our best configurations with state-of-the-art published results where available.

- We publish our source code as well as our ready-to-use checkpoints at: https://github.com/BOUALILILila/ExactMatchMarking

\section{Background and Related Work}

In this paper, we focus on ad hoc document retrieval (also referred to as document ranking) over corpora comprising either news articles or web pages. Following the standard formulation: Given a corpus of documents $C$, potentially large, the task of a ranking system is to produce a ranked list of $k$ documents from the corpus in response to a user's information need expressed as query $q$.

Deep Learning approaches have steadily grown in popularity since their introduction in IR over a decade ago. Even though Learning to Rank had reached its zenith early in the 2010s ( $\mathrm{Liu}, 2009, \mathrm{Li}, 2011)$, its use of discrete hand-crafted features, numbering in the hundreds or even more was a major limitation. The promise of Deep Learning models was precisely to obviate the need of such costly manual-engineered features by relying on neural networks and continuous vector representations. Soon, numerous neural ranking models emerged, such as DRMM (Guo et al., 2016), DUET (Mitra et al., 2017), KNRM (Xiong et al., 2017) and Conv-KNRM (Dai et al., 2018). We do not have sufficient space to thoroughly review early neural ranking models and therefore refer the readers to existing overviews (Mitra et al., 2018, Onal et al., 2018). Aside from the models that were specifically designed for document ranking, models from the NLP community built for semantic similarity share some architectural similarities and there has been cross-fertilization between NLP and IR (Yates et al., 2021). This interaction lead IR researchers to realise that relevance matching and semantic matching (e.g: sentence similarity) are different tasks (Guo et al., 2016). While the former requires proper handling of the exact matching signals, the later requires accurately capturing semantics. Thus, neural ranking models required new architecture designs to handle both semantic and exact matching signals. In (Mitra et al., 2017), authors proposed a duet architecture composed of two deep neural networks, a local model that captures exact matching signals and a distributed model for semantic matching. Despite the reported successes of these neural models, there has recently been some skepticism about whether these successes, in the absence of large 
amounts of data, are not just inflated by comparison to weak baselines. The study conducted over a 100 papers by (Yang et al. 2019a) on the Robust04 dataset showed that most models failed against strong non-neural baselines (RM3(Lavrenko and Croft, 2001)).

Recently, the inception of the transformer architecture (Vaswani et al. 2017) instigated a new wave of approaches (Nogueira and Cho, 2019; MacAvaney et al. 2019, Akkalyoncu Yilmaz et al., 2019) that, at last, were able to significantly outperform well-tuned traditional IR baselines such as RM3 (Lavrenko and Croft, 2001). Nogueira and Cho (Nogueira and Cho, 2019) describe the first successful application of BERT (Devlin et al., 2019) - known as monoBERT - to passage reranking where the ranking task is modeled as a binary classification problem over individual candidate passages. This work marks the beginning of the "BERT revolution". The results of the TREC Deep Learning Track 2019 (Craswell et al., 2020) demonstrated clearly the effectiveness of BERT-based models and revealed a significant distinction with the pre-BERT models. Regardless of its effectiveness, BERT has a key limitation for document ranking: it cannot handle long input sequences that are longer than 512 tokens. In order to address this challenge, Yang et al. (2019b) apply inference on sentences individually, and then use interpolation of the original document score - obtained by a traditional ranker - and the weighted top $n$ sentence scores to rerank the documents. Following the same strategy, Birch (Akkalyoncu Yilmaz et al. 2019) reports state-of-the-art effectiveness on the classic TREC newswire test collections Robust04, Core17 and Core18 using fine-tuned BERT on exclusively out of domain passage-level datasets (TREC Microblog, MS MARCO and TREC CAR). Their experiments demonstrate that relevance models can be transferred across different domains, which solves the problem of the lack of passage-level relevance annotations in the target domain. Similarly, Dai and Callan (2019) use passage-level evidence to fine-tune BERT by considering all passages from a relevant document as relevant. For inference, the document is split into overlapping passages and each passage is scored individually. Document scores based either on the score of the first passage, the best passages or the sum of all passage scores have been investigated and simple best passage score was found to be the best approach (BERT-MaxP). This was the first work to highlight BERT's capacity to exploit linguistically rich descriptions as opposed to previous keyword search techniques. MacAvaney et al. (2019) propose a new approach (CEDR) that incorporates the BERT's classification token [CLS] that encodes the representation of the full input into existing pre-BERT neural IR models. The authors show that this joint approach outperforms a vanilla BERT ranker. Instead of aggregating the scores of individual passages as in Birch and BERT-MaxP, Parade (Li et al., 2020) aggregates the passage representations. This yields an end-to-end differential model like CEDR but without the use of pre-BERT models. In order to obtain the document representation, several aggregation methods were investigated and using a small stack of transformer encoders was found to be the best method. Arguing that exact matching is a valuable cue for ranking, Boualili et al. (2020) propose a new adaptation 
of BERT, entitled MarkedBERT, that uses a marking technique to highlight exact match signals in the input sequence. The authors demonstrate the effectiveness of MarkedBERT on the MS MARCO passage ranking task and confirmed that marker tokens bring focus on exact matching terms through attention analysis. Beyond BERT, Nogueira et al. (2020) report new state-ofthe-art effectiveness on Robust04 using a novel adaptation of the pretrained sequence-to-sequence model T5 (Raffel et al., 2020) to the document ranking task. This new generation-based approach proved to be more efficient than BERT in the data-poor regime with limited training data. For an exhaustive review of all research lines using BERT-like models including elaborate multistage reranking architectures (Nogueira et al., 2019, Pradeep et al., 2021), efficiency-focused models (Hofstätter et al., 2020) and representation learning (MacAvaney et al., 2020b; Khattab and Zaharia, 2020), we refer readers to a recent survey (Yates et al., 2021).

In the light of the improvements brought by BERT to a wide range of IR tasks, many researchers investigate the reasons behind such substantial improvements. Padigela et al. (2019) empirically study a set of hypotheses that show that BM25 is more biased towards high query term frequency which hurts its performance while BERT retrieves passages with more novel words. However, they found that BERT fails at capturing the query context for long queries. Dai and Callan (2019) demonstrate that unlike traditional IR models, BERT takes advantage of stop words and punctuation thanks to its capacity to model language structure. Qiao et al. (2019) show that BERT is an interactionbased model (Guo et al. 2016), its advantage lies in the cross query-document attentions. Discarding these cross sequence interactions lead to a performance close to random. They also find that BERT assigns extreme matching scores to query-document pairs and most pairs get either one or zero ranking scores, showing it is well tuned by pre-training on large corpora. Câmara and Hauff (2020) analyze BERT using diagnostic datasets built from retrieval heuristics (Rennings et al., 2019). Their experiments show that BERT does not fulfil most retrieval heuristics created by IR experts and argue that these axioms are not suitable to understand BERT performance. MacAvaney et al. (2020a) introduce ABNIRML a new framework for analysing the behavior of neural IR models. The authors found that neural ranking models have fundamentally different characteristics from prior ranking models such as high sensitivity to word order and increasing relevance scores when non-relevant content is added to the document.

The work we present in this paper represents, to the best of our knowledge, the first paper detailing with a general approach of highlighting exact matching signals to enhance contextualized pretrained language models such as BERT and reporting an exhaustive set of experiments using long-document ranking benchmarks. Although, using a marking technique to emphasize exact term matches in the query-document pair was first proposed in our own previous work entitled MarkedBERT (Boualili et al. 2020) that represents our initial study. This last was limited to one marking technique on a passage ranking task. Aside from MarkedBERT, marking techniques were mentioned in the de- 
scriptions of our TREC-COVID challenge (Voorhees et al., 2021) submissions. This present work is a generalisation of the approach followed in MarkedBERT where we present a complete description of our ideas, comprehensive evaluation results and comparisons to state-of-the-art models on traditional ad hoc retrieval benchmarks.

\section{Augmenting pretrained Contextualized Language Models with Exact Match Signals}

In this section, we first describe the general BERT model architecture we adopt in this work and then present the marking strategies we propose to explicitly highlight exact term matches in the query-document pairs before feeding them to the BERT model. We consider the traditional formulation of exact matching where two terms $t_{1}$ and $t_{2}$ match exactly if their stems are identical. We use the Porter algorithm for stemming and stop words are not considered during marking. By adding explicit indications of exact matching signals in the textual inputs, the models can benefit from this traditional hint and adapt better to the ad hoc task.

\subsection{BERT model architecture}

BERT (Devlin et al. 2019) is a multi-layer bidirectional transformer encoder based on the original implementation described by Vaswani et al. (2017). It is pretrained on a large corpus of unlabeled data using two unsupervised tasks: Masked Language Model (MLM) and Next Sentence Prediction (NSP). BERT relies on a fine-tuning approach where minimal task specific parameters are fine-tuned together with the pretrained parameters for the downstream task. The special classification token [CLS] added to the beginning of the input is used for making judgements about sentence pairs. BERT is pretrained on the NSP task to predict whether Segment $A$ immediately precedes Segment $B$ in the original text. It can be further fine-tuned inexpensively on a downstream task.

We adopt the model configuration described by Nogueira and Cho (2019) referred to as monoBERT or vanilla BERT. In this configuration, the authors propose to apply BERT as a binary relevance classifier for text ranking. The architecture of the model is shown in Figure 1 . Using the same notation as Devlin et al. (2019), the query $q$ is fed as Segment $A$ and the candidate document $d$ as Segment $B$. A special token [CLS] is prepended to the input sequence, and a special delimiter token [SEP] is placed at the beginning and end of the document segment to build the input sequence $S$ as follows:

$$
S=[[C L S], Q,[S E P], D,[S E P]]
$$

where $Q$ and $D$ represent the sequences of tokens obtained after applying the WordPiece tokenizer to the query $q$ and document $d$ texts, respectively. 


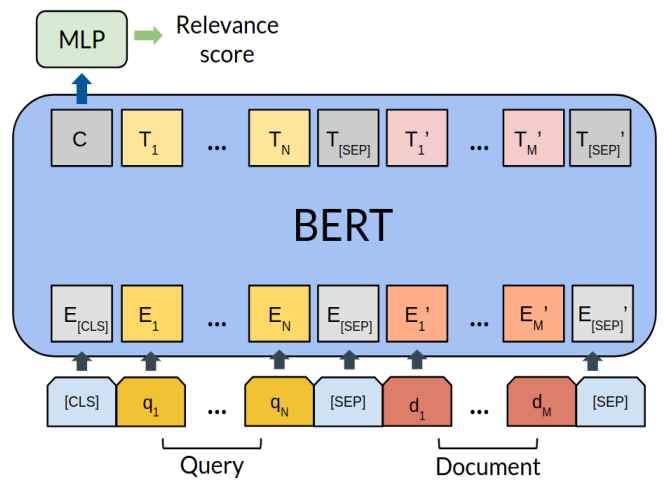

Fig. 1: BERT sentence pair classification architecture (Devlin et al. 2019) used in vanilla BERT - monoBERT (Nogueira and Cho, 2019).

Once the sequence $S$ is passed through BERT, the final vector representation $C$ of the standard classification token [CLS], that captures the interaction between the query and the document, is used as input to a single layer neural network that estimates a score score $R(d, q)$ quantifying how relevant the candidate document $d$ is to the query $q$. That is:

$$
R(d, q)=P(\text { Relevant }=1 \mid q, q)
$$

The details of the fine-tuning and inference process are given in section 4

\subsection{Excat match marking}

We propose different marking strategies that only intervene at the textual input level to augment the input sequence $S$ defined in Equation 1. Instead of altering the model's architecture in order to integrate the desired traditional signal, we prefer letting the model learn how to use the given hints and avoid the risk of introducing a systematic bias towards exact term matching. We introduce two types of marker tokens and investigate two levels of marking: Document Marking and Pair Marking. Table 3 illustrates examples of the two strategies combined with the two types of markers. Note that the Pre-Pair strategy corresponds to the strategy used in the MarkedBERT model (Boualili et al. 2020).

\subsubsection{Marker Tokens}

We investigate two types of marker tokens: The first consists of using a simple unique marker (\#) for all terms. The second uses precise markers consisting of newly introduced tokens $\left[e_{k}\right]$ and $\left[/ e_{k}\right]$, where $k=\{1, \ldots,|Q|\}$ identify query terms, that mark the start and the end of each matched term, respectively. 
Table 3: Example of the proposed marking strategies applied to the query $\mathbf{Q}$ : "causes of left ventricular hypertrophy", and the document D: "Left ventricular hypertrophy can occur when some factor ...".

\begin{tabular}{|c|c|c|c|}
\hline Marker & Level & Strategy & Marked Pair \\
\hline \multirow[t]{2}{*}{ Simple } & Document & Sim-Doc & $\begin{array}{l}\text { Q: causes of left ventricular hypertrophy } \\
\text { D: \#Left\# \#ventricular\# \#hypertro- } \\
\text { phy\# can occur... }\end{array}$ \\
\hline & Pair & Sim-Pair & $\begin{array}{l}\text { Q: causes of \#left\# \#ventricular\# \#hy- } \\
\text { pertrophy\# } \\
\text { D: \#Left\# \#ventricular\# \#hypertro- } \\
\text { phy\# can occur... }\end{array}$ \\
\hline \multirow[t]{2}{*}{ Precise } & Document & Pre-Doc & $\begin{array}{l}\text { Q: causes of left ventricular hypertrophy } \\
\text { D: } \quad[\mathrm{e} 2] \text { Left }[/ \mathrm{e} 2] \quad[\mathrm{e} 3] \text { ventricular[/e3] } \\
{[\mathrm{e} 4] \text { hypertrophy[/e4] can occur... }}\end{array}$ \\
\hline & Pair & Pre-Pair & 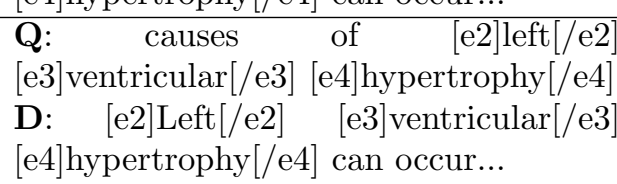 \\
\hline
\end{tabular}

This marking technique associates each unique query-term $q_{k}$ with a unique pair of marker tokens $\left[e_{k}\right]$ and $\left[/ e_{k}\right]$ that identifies it. If a term is repeated in the query, all occurrences of this query term will be highlighted using the same identifier i.e that of the first occurrence.

\subsubsection{Marking Level}

In order to better understand whether it is relevant to mark both the query and the document segments or the document segment only, we investigate two marking levels: Document and Pair marking. In the former, the occurrences of query terms in the document are marked in the document segment while in the later, the exact matching terms are marked in both the document and query segments as shown in Table 3. We use the same notations defined in the model's architecture where $Q$ refers to the query segment and $D$ refers to the document segment that constitute the input sequence $S$.

Document marking. It only augments the document segment $D$ with the marker tokens that mark the start and the end of each occurrence of query terms in the document. Considering a query $Q=\left\{q_{1}, \ldots, q_{|Q|}\right\}$ and a document $D=\left\{d_{1}, \ldots, d_{|D|}\right\}$, if $\left\{d_{i}, d_{j}\right\}$ are occurrences of query term $q_{n}$ and $d_{l}$ is the only occurrence of $q_{m}$ in $D$ with $1<n<m<|Q|$ and $1<i<j<l<|D|$, the augmented query and document sequences $\tilde{Q}$ and $\tilde{D}$, respectively, are as 
follows when using the simple markers:

$$
\begin{aligned}
\tilde{Q} & =\left\{q_{1}, \ldots, q_{n}, \ldots, q_{m}, \ldots, q_{|Q|}\right\} \\
\tilde{D} & =\left\{d_{1}, \ldots, \# d_{i} \#, \ldots, \# d_{j} \#, \ldots, \# d_{l} \#, \ldots, d_{|D|}\right\}
\end{aligned}
$$

and as follows when using the precise markers:

$$
\begin{aligned}
\tilde{Q} & =\left\{q_{1}, \ldots, q_{n}, \ldots, q_{m}, \ldots, q_{|Q|}\right\} \\
\tilde{D} & =\left\{d_{1}, \ldots,\left[e_{n}\right] d_{i}\left[/ e_{n}\right], \ldots,\left[e_{n}\right] d_{j}\left[/ e_{n}\right], \ldots,\left[e_{m}\right] d_{l}\left[/ e_{m}\right], \ldots, d_{|D|}\right\}
\end{aligned}
$$

Pair marking. It augments both the query and document sequences with the special tokens that mark the start and the end of each exact matched term between the query and the document. In our experiments, a query term with no occurrences in the document is not marked. Considering the same example as in the Document marking strategy, the augmented query and document sequences $\tilde{Q}$ and $\tilde{D}$, respectively, are as follows:

$$
\begin{aligned}
\tilde{Q} & =\left\{q_{1}, \ldots, \# q_{n} \#, \ldots, \# q_{m} \#, \ldots, q_{|Q|}\right\} \\
\tilde{D} & =\left\{d_{1}, \ldots, \# d_{i} \#, \ldots, \# d_{j} \#, \ldots, \# d_{l} \#, \ldots, d_{|D|}\right\}
\end{aligned}
$$

when using the simple markers, or as follows when using the precise markers:

$$
\begin{aligned}
\tilde{Q} & =\left\{q_{1}, \ldots,\left[e_{n}\right] q_{n}\left[/ e_{n}\right], \ldots,\left[e_{m}\right] q_{m}\left[/ e_{m}\right], \ldots, q_{|Q|}\right\} \\
\tilde{D} & =\left\{d_{1}, \ldots,\left[e_{n}\right] d_{i}\left[/ e_{n}\right], \ldots,\left[e_{n}\right] d_{j}\left[/ e_{n}\right], \ldots,\left[e_{m}\right] d_{l}\left[/ e_{m}\right], \ldots, d_{|D|}\right\}
\end{aligned}
$$

\section{Experimental setup}

This section presents the experimental setup used for studying the effectiveness of our models including datasets, baselines, fine-tuning and inference settings.

\subsection{Datasets}

We conduct experiments on two standard ad-hoc benchmarks: Robust04 and GOV2. Robust04 ${ }^{1}$ is a news wire collection comprising $500 K$ documents (TREC Disks 4 and 5) and 249 judged topics. Each topic is composed of three fields: The "title" is a short keyword query, the "description" is a longer wellformed natural language sentence that describes the information need and the "narrative" is a paragraph that provides guidance for relevance assessment. Table 4 provides an example of a TREC Robust04 topic. GOV2 ${ }^{2}$ is a Web collection crawled from government Websites in early 2004 comprising $25 \mathrm{M}$ documents and only 149 topics in the same format as Robust04 topics with

\footnotetext{
1 https://trec.nist.gov/data/robust/04.guidelines.html

2 http://ir.dcs.gla.ac.uk/test_collections/gov2-summary.htm
} 
Table 4: Example of Robust04 search topic: Topic 302.

\begin{tabular}{ll}
\hline Title & Poliomyelitis and Post-Polio \\
Description & Is the disease of Poliomyelitis (polio) under control in the \\
& world? \\
Narrative & $\begin{array}{l}\text { Relevant documents should contain data or outbreaks of the } \\
\text { polio disease (large or small scale), medical protection against }\end{array}$ \\
& the disease, reports on what has been labeled as "post-polio" \\
& problems. Of interest would be location of the cases, how \\
& severe, as well as what is being done in the "post-polio" area. \\
\hline
\end{tabular}

Table 5: Benchmarks statistics.

\begin{tabular}{lccc}
\hline Benchmark & \# Judged Topics & \# Documents & \# Words per Document \\
\hline Robust04 & 249 & $0.5 M$ & $0.738 K$ \\
GOV2 & 149 & $25 M$ & $3.768 K$ \\
\hline
\end{tabular}

title, description and narrative. Documents in the GOV2 corpus are on average much longer than those in the Robust04 corpus. Table 5 resumes some statistics on the two collections. As we focus only on reranking, the reported average document length is obtained from the top 1,000 documents returned by BM25.

\subsection{Baselines}

We compare our models against the following baselines.

\subsubsection{Traditional non-neural baselines}

BM25, we use the Anserini (Yang et al., 2017) implementation with default parameters. For description queries, we set $k_{1}=0.9$ for Robust04 and $k_{1}=2.0$ for GOV2 and $b=0.6$ for both datasets. This unsupervised model serves both as a baseline and as the first stage retriever in all our experiments.

$B M 25+R M 3$, a query expansion model based on RM3 (Lavrenko and Croft, 2001) considered as a strong non-neural baseline. We use the Anserini (Yang et al. 2017) implementation with the default parameters. For description queries, we use 20 expansion terms following (Li et al., 2020).

\subsubsection{Vanilla baseline}

The vanilla model is our main baseline since it represents the core model we augment with explicit exact match cues in our proposed models. The vanilla 
baseline and our models share the same configuration and evaluation setup making it suitable for a fair comparison.

\subsubsection{State of the art baselines}

In addition to the vanilla baseline, we conduct a comparative evaluation with Birch (Akkalyoncu Yilmaz et al. 2019), BERT-MaxP (Dai and Callan, 2019), Parade (Li et al. 2020) and T5 (Nogueira et al. 2020), which are the transformerbased models representing the state of the art, in section 5.5

\subsection{Training}

We use the base version (12 layers, 768 hidden size, 12 heads, 110M parameters) of BERT due to hardware limitations. We fine-tune both our vanilla baseline and our models augmented with the different marking strategies on the large publicly released MS MARCO passage dataset. We use a batch-size of 128 and the maximum sequence length (128 sequences $\times 512$ tokens $=$ 65,536 tokens/batch) for $100 k$ on free Google Colab TPU $3^{3}$ We use Adam optimizer (Kingma and $\mathrm{Ba}, 2015)$ with the initial learning rate set to $3 e^{-6}$ and linear decay of the learning rate. The drop out rate is set to 0.1 for all our experiments. We use the open source implementation of BERT by Hugging Face (Wolf et al. 2020). It is important to note that fine-tuning an augmented model with a marking strategy does not add a computational cost compared to the vanilla model.

\subsection{Inference}

We use a two-stage ranking pipeline. We retrieve an initial candidate list of top 1,000 documents per query using BM25. We use the BM25 implementation from off-the-shelf Anserini open-source IR toolkit (Yang et al., 2017).

The length of BERT's input sequence cannot exceed 512 tokens due to the fact that the positional embeddings were trained on sequences of a maximum length of 512 tokens. This limitation prevents from directly applying our models to long documents. Following the strategy proposed by Dai and Callan (Dai and Callan, 2019), we split each document into overlapping passages that can be handled individually by BERT. Passages are generated using a sliding window of 150 words and a stride of 75 words, formally expressed as $d=\left\{p_{1}, \ldots, p_{n}\right\}$ where $n$ is the number of passages in the document $d$. As a trade-off between latency and effectiveness, we only consider a maximum of 30 passages per document. The first and last passages are always picked while the remaining 28 are randomly chosen. The fine-tuned BERT models on exclusively out-of-domain data are used afterwards to predict the relevance of

3 https://colab.research.google.com 
each passage w.r.t a query $q$ independently. The best scoring passage is then taken as a proxy for the Document-level relevance:

$$
R(d, q)=\max \left(R\left(p_{1}, q\right), \ldots, R\left(p_{n}, q\right)\right)
$$

For the queries we consider both the topic titles that are preferred by most pre-BERT models including BM25, and the descriptions that are more similar to MS MARCO's natural language questions.

\section{Results and Analysis}

We empirically evaluate the contribution of our marking strategies compared to the vanilla baseline in different experimental scenarios. We use standard IR collections issued from TREC evaluation campaign $4^{4}$ and discuss the following research questions:

- RQ1: Is exact match marking beneficial to pretrained transformers exemplified by BERT?

- RQ2: Do exact match scores from the first-stage retriever contribute to end-to-end effectiveness of the pretrained transformers and how exact match marking affects this contribution?

- RQ3: At which phase the exact match marking is the most beneficial in a multi-phase fine-tuning configuration?

- RQ4: Is exact match marking beneficial in alternative transformer-based models such as ELECTRA?

\subsection{Performance of the models augmented with exact match marking}

We evaluate the contribution of our proposed exact match marking strategies and discuss our research question RQ1 Is exact match marking beneficial to pretrained transformers exemplified by BERT? by comparing the augmented models with exact match marking to the vanilla baseline. We use the fine-tuned models on exclusively out-of-domain data (MS MARCO passage collection) to rerank the documents retrieved by BM25 in the first-stage. We do not train the models on the target collections (Robust04, GOV2), we use all their queries and relevance judgements as a held-out test set. Thus, this evaluation is an instance of a zero-shot transfer setting. Table 6 shows the reranking effectiveness of our different models and baselines on the top 1,000 candidate documents retrieved by BM25 from Robust04 and GOV2 collections using both the title and description fields of the TREC topics. We recall that titles are short key word queries preferred by traditional bag-of-words models like BM25 and descriptions are well-written natural language queries similar to MS MARCO's questions on which the BERT models are fine-tuned.

4 trec.nist.gov 
Table 6: Reranking effectiveness in the zero-shot transfer setting of the different models on Robust04 and GOV2 collections. Best performance is highlighted in bold. Significant improvements over the vanilla baseline with $p<0.05$ and $p<0.01$ after Bonferroni correction are indicated with $\dagger$ and $\ddagger$ respectively. Significant inferiority with $p<0.05$ is marked with $*$. For each measure, the improvement rate over the vanilla baseline is given (\%).

\begin{tabular}{|c|c|c|c|c|c|c|c|c|}
\hline Robust04 & \multicolumn{4}{|c|}{ Title run } & \multicolumn{4}{|c|}{ Description run } \\
\hline Model & nDCG@2 & & $\mathrm{P} @ 20$ & & nDCG@2 & & $\mathrm{P} @ 20$ & \\
\hline BM25 & 0.4240 & - & 0.3631 & - & 0.4058 & - & 0.3345 & - \\
\hline BM25+RM3 & 0.4407 & - & 0.3821 & - & 0.4255 & - & 0.3661 & - \\
\hline Vanilla BERT & 0.4652 & - & 0.4046 & - & 0.4510 & - & 0.3851 & - \\
\hline Sim-Doc BERT & $0.4447^{*}$ & $-4.4 \%$ & $0.3831^{*}$ & $-5.3 \%$ & $0.4166^{*}$ & $-7.6 \%$ & $0.3510^{*}$ & $-8.9 \%$ \\
\hline Sim-Pair BERT & 0.4773 & $+2.6 \%$ & 0.4155 & $+2.7 \%$ & $0.4931^{\ddagger}$ & $+9.3 \%$ & $0.4169^{\ddagger}$ & $+8.3 \%$ \\
\hline Pre-Doc BerT & 0.4767 & $+2.5 \%$ & 0.4084 & $+0.9 \%$ & $0.4789^{\ddagger}$ & $+6.2 \%$ & $0.4026^{\ddagger}$ & $+4.5 \%$ \\
\hline Pre-Pair BERT & 0.4654 & $+0.0 \%$ & 0.4024 & $-0.5 \%$ & $0.4795^{\ddagger}$ & $+6.3 \%$ & $0.4034^{\ddagger}$ & $+4.8 \%$ \\
\hline GOV2 & \multicolumn{4}{|c|}{ Title run } & \multicolumn{4}{|c|}{ Description run } \\
\hline Model & nDCG@2 & & $\mathrm{P} @ 20$ & & nDCG@2 & & $\mathrm{P} @ 20$ & \\
\hline BM25 & 0.4774 & - & 0.5362 & - & 0.4264 & - & 0.4705 & - \\
\hline BM25+RM3 & 0.4851 & - & 0.5634 & - & 0.4212 & - & 0.4966 & - \\
\hline Vanilla BERT & 0.4533 & - & 0.5272 & - & 0.4696 & - & 0.5248 & - \\
\hline Sim-Doc BERT & 0.4588 & $+1.2 \%$ & 0.5349 & $+1.5 \%$ & 0.4686 & $-0.2 \%$ & 0.5262 & $+0.3 \%$ \\
\hline Sim-Pair BERT & 0.4468 & $-1.4 \%$ & 0.5134 & $-2.6 \%$ & 0.4687 & $-0.2 \%$ & 0.5326 & $+1.5 \%$ \\
\hline Pre-Doc BerT & 0.4485 & $-1.1 \%$ & 0.5121 & $-2.9 \%$ & 0.4768 & $+1.5 \%$ & 0.5315 & $+1.3 \%$ \\
\hline Pre-Pair BERT & 0.4515 & $-0.4 \%$ & 0.5238 & $-0.6 \%$ & 0.4752 & $+1.2 \%$ & 0.5285 & $+0.7 \%$ \\
\hline
\end{tabular}

Impact of the marker type and marking level on the performance. On Robust04, marking both the query and the document -models based on pair marking- has more impact on the simple marker than the precise marker. On the description queries, Sim-Pair BERT achieves a nDCG@20 of 0.4931 while Sim-Doc BERT has an nDCG@20 of only 0.4166, and achieve 0.4773 compared to 0.4447 , respectively, on title queries. While the marking strategy has a lower impact on models using the precise markers (Pre-Doc BERT and Pre-Pair BERT) especially on descriptions. On the other hand, results on the GOV2 collection are quite mitigated.

Marking both the query and the document segments with a simple marker (\#) appears to be the best setting, Sim-Pair BERT has the best ranking accuracy among the four strategies tested with clear margins on the Robust04 collection especially on descriptions.

Comparison with baselines. All BERT-based models achieve substantially better performance on both collection compared to the traditional nonneural baselines at the only exception of GOV2 titles. We observe a discrepancy in the impact of the exact match marking on GOV2 compared to Robust04. While all our models, except Sim-Doc BERT, significantly outperform the vanilla baseline on Robust04 descriptions or at least achieve similar performance on titles, our models have no significant impact on GOV2. Importantly, in no case a marking-based model leads to a significant degradation of performance on GOV2. The disparity in the behavior of the models on the two benchmarks is probably due to the nature of the documents involved. 
Table 7: Recall of BM25 on Robsut04 and GOV2 collections on both title and description queries.

\begin{tabular}{l|cc}
\hline Collection & Title & Description \\
\hline Robust04 & 0.6989 & 0.6519 \\
\hline GOV2 & 0.7106 & 0.6024 \\
\hline
\end{tabular}

While Robust04 comprises well-written news articles, GOV2 documents are web pages that include navigation bars, advertisements, tables and discontinuous text. The zero-shot domain transfer -from the MS MARCO fine-tuned models to Robust04 articles- seems to be more attainable than to GOV2 web pages even though MS MARCO passages were extracted from the web. We hypothesise that further fine-tuning on domain-specific data may be required to learn better domain-specific text representations. We investigate this indomain adaptation in section 5.3

Since exact match marking is not yet fully effective on the GOV2 collection, we rely on the results obtained on Robust04 to conclude that the most effective yet simple marking strategy is Sim-Pair BERT that will be confirmed for GOV2 in later experiments. We choose to continue our analysis using the Sim-Pair BERT strategy for more clarity, the full results using all the marking strategies can be found in Appendix A

Title vs. description queries. Since we are in a reranking configuration, it is important to note that the first stage retriever BM25, as most pre-BERT ranking models, prefers short key word queries to longer natural language descriptions (Dai and Callan, 2019, Nogueira et al., 2020). Table 7 shows the recall at rank 1,000 of BM25 for both title and description queries, where we notice a substantial difference in recall affecting the quality of the candidate documents that the reranking models receive. Despite this disadvantageous initialization, the reranking models manage to reduce the gap between title and description runs. The improvement rate over BM25 is much higher for description queries compared to title queries on both collections especially on GOV2 where vanilla BERT has a change rate of $-5.0 \%$ over BM25, while it achieves over $+10 \%$ gain on descriptions. The descriptions that are longer natural language queries carrying richer information that could not be fully harnessed by the traditional bag-of-words method, are more effectively leveraged in the reranking stage. This BERT ability was already noted in previous work (Dai and Callan, 2019), and Sim-Pair BERT follows the same preference, as it improves the search accuracy of the description runs more effectively than the title runs. The overall performance reported for our model using descriptions clearly surpasses that obtained using titles by $+4.1 \%$ on average, despite the lower recall in the initial stage.

Impact of the initial stage retriever. Considering that first stage ranker BM25 has higher recall on title queries, and that the marking-based models prefer description queries, we propose a hybrid reranking pipeline where the documents retrieved by BM25 using title queries are reranked with 
Table 8: Reranking effectiveness in the zero-shot transfer setting of the different models on Robust04 and GOV2 collections using the hybrid pipeline.Best performance is highlighted in bold. Significant improvements over the vanilla baseline with $p<0.05$ and $p<0.01$ after Bonferroni correction are indicated with $\dagger$ and $\ddagger$ respectively. Significant inferiority with $p<0.05$ is marked with *. For each measure, the improvement rate over the vanilla baseline is given $(\%)$.

\begin{tabular}{l|lllc|lccc}
\hline & \multicolumn{3}{|c|}{ Robust04 } & \multicolumn{4}{c}{ GOV2 } \\
\hline Model & nDCG@20 & \multicolumn{3}{|c|}{ P@20 } & & nDCG@20 & P@20 & \\
\hline BM25 & 0.4240 & - & 0.3631 & - & 0.4774 & - & 0.5362 & - \\
BM25+RM3 & 0.4407 & - & 0.3821 & - & 0.4851 & - & 0.5634 & - \\
\hline Vanilla BERT & 0.4845 & - & 0.4147 & - & 0.4937 & - & 0.5611 & - \\
Sim-Pair BERT & $\mathbf{0 . 5 2 3 9}^{\ddagger}$ & $+8.1 \%$ & $\mathbf{0 . 4 4 4 6} 6^{\ddagger}$ & $+7.2 \%$ & $\mathbf{0 . 4 9 9 1}$ & $+1.1 \%$ & $\mathbf{0 . 5 6 9 5}$ & $+1.5 \%$ \\
\hline
\end{tabular}

the BERT-based models using the description queries. Using this hybrid pipeline allow as to obtain a higher recall in the first stage since BM25 performs better on short keyword queries, and thus better candidate documents for reranking. Description queries are longer statements of information needs more suitable for pretrained reranking models to fulfill their potential. This pipeline remains realistic as language queries may be generated from standard key-word queries (Padaki et al., 2020). This hybrid approach is also adopted in recent state-ofthe-art ranking model based on T5(Nogueira et al., 2020).

Table 8 shows the results obtained using the hybrid reranking pipeline on both test collections. Unsurprisingly, using better candidate documents for reranking with descriptions yields even better accuracy. The vanilla BERT model achieves an improvement rate of $+14 \%$ over BM25 on Robust0 4 and $+3.4 \%$ on GOV2 (we recall that BM25 results are obtained using titles). Adding exact match marking in the hybrid reranking pipeline outperforms the vanilla baseline on both collections; Significantly on Robust04 with a gain of over $+8 \%$.

5.2 Contribution of the first-stage retriever scores to the end-to-end effectiveness

Our experimental design is based on a two-stage ranking architecture also known as a retrieve-then-rerank architecture where our BERT-based models rerank the documents retrieved by the BM25 model. In this section we evaluate the contribution of the best match scores from the initial bag-ofwords retriever to the end-to-end effectiveness by simply combining BM25's document-level scores with the passage-level evidence from the reranker using linear interpolation. We follow the linear combination defined in the Birch model (Akkalyoncu Yilmaz et al. 2019).

Birch uses a monoBERT sentence-level relevance classifier at its core. To determine document relevance $s_{f}$, inference is applied over each individual sentence $s_{i}$ in a candidate document $d$, and then the top $n$ sentence scores 
are combined with the original document score $s_{d o c}$ given by the first-stage retriever as follows:

$$
s_{f}=\alpha . s_{d o c}+(1-\alpha) \cdot \sum_{i=1}^{n} w_{i} . s_{i}
$$

where $s_{i}$ is the $i$-th top scoring sentence according to monoBERT. The parameters $\alpha$ and $w_{i}$ 's are tuned via cross-validation. In other words, the relevance score of a document comes from the combination of its document-level term-matching score and evidence contributions from the top sentences in the documents as determined by monoBERT.

For our experiments, the linear interpolation is applied to the results obtained in the zero-shot transfer setting with the best-scoring passage $(n=1)$. In other words, we use the score combination defined in Equation 4 on the document scores obtained by the BM25 retriever at cutoff 1,000 and their corresponding scores estimated with the best-scoring passage method by the reranking models. Table 9 first shows the results of the traditional BM25 model alone, then the second and third sections are each dedicated to a reranker: vanilla and Sim-Pair BERT models. For both rerankers, we remind the results of the model alone obtained in the zero-shot transfer setting and then present the end-to-end effectiveness after interpolating BM25 scores (+ BM25) with the indication of the change rate (\%) over the reranker-only effectiveness. These results allow us to answer our research question RQ2 Do exact match scores from the first-stage retriever contribute to end-to-end effectiveness of the pretrained transformers and how exact match marking affects this contribution?

Impact of interpolating BM25 scores. Interpolating BM25 scores (Best Match) that are solely based on surface-level features such as TF and IDF leads to a significant gain in performance, indicating that BM25 documentlevel scores provide an additional relevance signal that the BERT-based models alone could not effectively capture. We notice that the improvement rate resulting from interpolating BM25 scores is much substantial on the GOV2 collection ( $+15 \%$ in average) compared to Robust04 $(+5.7 \%$ in average). The fact that the BERT models outperform BM25 by a large margin on Robust04 while this margin is much smaller on the GOV2 can explain why BM25 scores have more incidence on the end-to-end effectiveness on GOV2 than on Robust04.

Impact of exact match marking. From Table 9, we can clearly see that for Robust04, where the exact match marking is effective, the improvement rate over the reranker-only effectiveness is lower when using exact match marking, about $+12 \%$ in average, compared to the vanilla model with $+22 \%$ gain in average. In other words, the impact of the BM25 scores is more important on the vanilla model compared to the Sim-Pair model. While on GOV2 the improvement rate after BM25 scores interpolation compared to the reranker-only performance is either comparable or slightly higher when using exact match marking compared to the vanilla baseline. However, the performance of the 
Table 9: Reranking effectiveness of the different models before and after interpolating BM25 scores on Robust04 and GOV2 collections. Best performance is highlighted in bold. For each measure, the improvement rate over the reranking performance without BM25 scores interpolation is given (\%).

\begin{tabular}{|c|c|c|c|c|c|c|c|c|c|c|c|c|}
\hline Robust04 & \multicolumn{4}{|c|}{ Title run } & \multicolumn{4}{|c|}{ Description run } & \multicolumn{4}{|c|}{ Hybrid run } \\
\hline Model & nDCG@: & & $P @ 20$ & & nDCG@2 & & $\mathrm{P} @ 20$ & & nDCG@2 & 0 & $P @ 20$ & \\
\hline BM25 & 0.4240 & - & 0.3631 & - & 0.4058 & - & 0.3345 & - & 0.4240 & - & 0.3631 & - \\
\hline Vanilla BERT & 0.4652 & 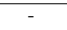 & 0.4046 & - & 0.4510 & & 0.3851 & & 0.4845 & & 0.4147 & \\
\hline + BM25 & 0.4932 & $+6.0 \%$ & 0.4255 & $+5.2 \%$ & 0.4856 & $+7.7 \%$ & 0.4062 & $+5.5 \%$ & 0.5266 & $+8.7 \%$ & 0.4488 & $+8.2 \%$ \\
\hline $\begin{array}{c}\text { Sim-Pair BERT } \\
\text { + BM25 }\end{array}$ & $\begin{array}{l}0.4773 \\
\mathbf{0 . 4 9 4 7}\end{array}$ & $\begin{array}{r}- \\
+3.6 \%\end{array}$ & $\begin{array}{l}0.4155 \\
\mathbf{0 . 4 2 6 5}\end{array}$ & $\begin{array}{c}- \\
+2.6 \%\end{array}$ & $\begin{array}{l}0.4931 \\
\mathbf{0 . 5 0 9 8}\end{array}$ & $\begin{array}{r}- \\
+3.4 \%\end{array}$ & $\begin{array}{l}0.4169 \\
\mathbf{0 . 4 2 7 9}\end{array}$ & $\begin{array}{l}- \\
+2.6 \%\end{array}$ & $\begin{array}{l}0.5239 \\
\mathbf{0 . 5 4 9 7}\end{array}$ & $\begin{array}{r}- \\
+4.9 \%\end{array}$ & $\begin{array}{l}0.4446 \\
\mathbf{0 . 4 7 0 7}\end{array}$ & $\begin{array}{r}- \\
+5.9 \%\end{array}$ \\
\hline GOV2 & \multicolumn{4}{|c|}{ Title run } & \multicolumn{4}{|c|}{ Description run } & \multicolumn{4}{|c|}{ Hybrid run } \\
\hline Model & nDCG@: & & $\mathrm{P} @ 20$ & & nDCG@2 & & $\mathrm{P} @ 20$ & & nDCG@2 & & $\mathrm{P} @ 20$ & \\
\hline BM25 & 0.4774 & - & 0.5362 & - & 0.4264 & - & 0.4705 & - & 0.4774 & - & 0.5362 & - \\
\hline Vanilla BERT & 0.4533 & - & 0.5272 & - & 0.4696 & - & 0.5248 & - & 0.4937 & - & 0.5611 & - \\
\hline + BM25 & 0.5320 & $+17 . \%$ & 0.5987 & +13 & 0.5166 & $+10 . \%$ & 0.5742 & $+9.4 \%$ & 0.5722 & $+16 . \%$ & 0.6383 & $+14 \%$ \\
\hline $\begin{array}{l}\text { Sim-Pair BERT } \\
\text { + BM25 }\end{array}$ & $\begin{array}{l}0.4468 \\
\mathbf{0 . 5 3 2 7}\end{array}$ & $\begin{array}{r}-\overline{-} \\
+19 . \%\end{array}$ & $\begin{array}{l}0.5134 \\
\mathbf{0 . 6 0 0 0}\end{array}$ & $\begin{array}{l}- \\
+17 \%\end{array}$ & $\begin{array}{l}0.4687 \\
\mathbf{0 . 5 2 3 5}\end{array}$ & $\begin{array}{r}- \\
+12 . \%\end{array}$ & $\begin{array}{l}0.5326 \\
\mathbf{0 . 5 8 9 3}\end{array}$ & $+-\overline{11 . \%}$ & $\begin{array}{l}0.4991 \\
\mathbf{0 . 5 7 7 8}\end{array}$ & $\begin{array}{r}- \\
+16 . \%\end{array}$ & $\begin{array}{l}0.5695 \\
\mathbf{0 . 6 4 9 7}\end{array}$ & $\begin{array}{r}- \\
+14 . \%\end{array}$ \\
\hline
\end{tabular}

Sim-Pair BERT model with BM25 scores interpolation is, in all cases, higher than the vanilla BERT + BM25 performance regardless of the improvement rate brought by the score combination. Since we use the results obtained in the zero-shot domain transfer setting where, we recall, the exact the marking is more effective, the gains of the Sim-Pair BERT +BM25 configuration over the vanilla BERT+BM25 are more substantial on Robust04 than on GOV2.

Contribution of BM25 scores. The contribution of BM25 scores is controlled by the parameter $\alpha$ in Equation 4 which we tuned via 5 -fold incollection cross validation. In all scenarios, the weight put on $\alpha$ is non-negligible, in other words, the contribution of BM25 signals remain important, this observation was also reported for the Birch model (Yates et al., 2021). However, we notice that the weight of $\alpha$ is always lighter when combining with the SimPair BERT model that uses exact match marking. For Robust04 descriptions, the vanilla BERT + BM25 baseline puts a weight of $\alpha \in\{0.3,0.4\}$ on BM25 scores, when Sim-Pair BERT+BM25 only consider a contribution of $\alpha=0.2$ from BM25, while achieving substantially better performance. This indicates that the vanilla model relies more on BM25 to complete its relevance estimation unlike the marking-based model that is able to effectively capture more relevance signals and thus needing less contribution from BM25 scores.

Figure2 visualizes the end-to-end ranking accuracy measured by nDCG@20 for $\alpha \in[0,1]$ on both Robust04 and GOV2 collections. On Robust04, we can clearly see that Sim-Pair BERT + BM25 reaches the most effective combination with smaller contribution from BM25 scores (smaller $\alpha$ ), while the vanilla baseline requires more intervention from BM25 and still cannot reach the performance of Sim-Pair BERT +BM25, especially on descriptions. It is only logical that the most performing model, that outperforms BM25 by a large margin, requires less contribution from this later. Nevertheless, if we take the example of the GOV2 descriptions, despite the similar starting performance at $\alpha=0.0$ of vanilla and Sim-Pair BERT models, the gap between their performance starts getting wider at only $\alpha=0.1$ to reach its peak at $\alpha=0.2$. 


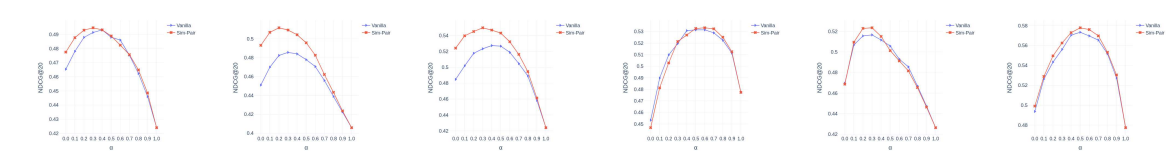

(a) Robust04 ti-(b) Robust04(c) Robust04(d) GOV2 titles(e) GOV2 de-(f) GOV2 hytles descrip. hybrid scrip. brid

Fig. 2: The end-to-end ranking accuracy of the vanilla BERT and Sim-Pair BERT models with BM25 scores interpolation on Robust04 and GOV2 collections. $\alpha=0.0$ indicates the reranking model effectiveness only without BM25 scores, and $\alpha=1.0$ means that only BM25 scores are used.

Combining the original document score obtained in the first-stage retriever with passage-level evidence from BERT-based reranking models to determine the final relevance score of a document yields substantial gains in performance. Relevance scores based on traditional IR axioms complete the relevance signals captured by contextual pretrained LMs such as BERT. Moreover, using our simple marking strategy to highlight the exact matching signals in the querydocument pairs enhance BERT's own ability to estimate relevance and thus, requires less contribution from BM25 to achieve the best performance.

\subsection{Multi-Phase Fine-Tuning}

In previous experiments, we leveraged out-of-domain relevance assessments to fine-tune our BERT models. This fine-tuning aims at providing the model with general notions of relevance matching. However, transferring these relevance patterns to the target corpus may, in some cases, be ineffective. To overcome this domain-transfer limitation, we use additional fine-tuning on labeled data drawn from the same distribution as the final task, in other words, in-domain labeled data fine-tuning. This approach is known as "stage-wise" or "multiphase" fine-tuning (Yates et al., 2021).

Once the models are fine-tuned on the MS MARCO passage dataset following the training setting described in section 4.3, we further fine-tune them on the target task using 5-fold cross validation for both Robust04 and GOV2 collections. We use the folds from Yang et al. (2019a) for Robust04 and the 5 -folds configuration adopted by Li et al. (2020).

Following prior work by Dai and Callan (Dai and Callan, 2019), we consider a maximum of 30 passages per document as a trade-off between latency and effectiveness. During training, passages issued from the top 1,000 documents retrieved by BM25 for queries in the training folds are sub-sampled to avoid catastrophic forgetting. Aside from the first passage, passages in a document are randomly preserved with a probability of 0.1 . Passages from a relevant document according to the ground-truth (TREC relevance judgements) are taken as positive examples and passages issued from the other remaining documents as negative examples. We use a pointwise cross entropy loss and fine-tune the 
Table 10: Reranking effectiveness in the multi-phase vs. zero-shot transfer setting for the Sim-Pair and vanilla models on Robust04 and GOV2 collections. Best performance is highlighted in bold. Significant improvements over the vanilla baseline with $p<0.05$ and $p<0.01$ after Bonferroni correction are indicated with $\dagger$ and $\ddagger$ respectively for the same setting. Significant inferiority with $p<0.05$ is marked with $*$. Change rate over the vanilla baseline in the same setting are reported for each metric (\%).

\begin{tabular}{|c|c|c|c|c|c|c|c|c|c|c|c|c|}
\hline \multirow{2}{*}{$\begin{array}{l}\text { Robust04 } \\
\text { Model }\end{array}$} & \multicolumn{4}{|c|}{ Title run } & \multicolumn{4}{|c|}{ Description run } & \multicolumn{4}{|c|}{ Hybrid run } \\
\hline & DCG@2 & & $\mathrm{P} @ 20$ & & $\mathrm{nDCG} @ 2$ & & $\mathrm{P} @ 20$ & & nDCG@2 & & $\mathrm{P} @ 20$ & \\
\hline & 0.4240 & - & 0.3631 & - & 0.4058 & - & 0.3345 & - & 0.4240 & - & 0.3631 & - \\
\hline BM25+RM3 & & - & & - & & - & & - & & - & & - \\
\hline \multicolumn{13}{|l|}{ Zero-shot transfer } \\
\hline $\begin{array}{l}\text { Vanilla BERT } \\
\text { Sim-Pair }\end{array}$ & & - & $\begin{array}{l}0.4096 \\
0.4129\end{array}$ & $\begin{array}{c}- \\
+0.8\end{array}$ & $\begin{array}{l}0.4611 \\
0.4923^{\ddagger}\end{array}$ & $\begin{array}{c}- \\
+6.8\end{array}$ & & $\begin{array}{c}- \\
+5.6\end{array}$ & & $\begin{array}{c}- \\
+5.7\end{array}$ & & - \\
\hline \multicolumn{13}{|l|}{ Multi-ph } \\
\hline $\begin{array}{l}\text { Vanilla }{ }_{B E R} \\
\text { Sim-Pair }\end{array}$ & $\begin{array}{l}0.4995 \\
\mathbf{0 . 5 0 5 8}\end{array}$ & $\begin{array}{r}- \\
+1.3 \%\end{array}$ & $\begin{array}{l}0.4275 \\
\mathbf{0 . 4 3 7 1}\end{array}$ & $\begin{array}{c}- \\
+2.2 \%\end{array}$ & $\begin{array}{l}0.5368 \\
\mathbf{0 . 5 4 7 9}^{\dagger}\end{array}$ & $\begin{array}{r}- \\
+2.1 \%\end{array}$ & $\begin{array}{l}0.4492 \\
\mathbf{0 . 4 5 7 4 ^ { \dagger }}\end{array}$ & $\begin{array}{r}- \\
+1.8 \%\end{array}$ & $\begin{array}{l}0.5546 \\
\mathbf{0 . 5 7 0 1}^{\ddagger}\end{array}$ & $\begin{array}{c}- \\
+2.8 \%\end{array}$ & $\begin{array}{l}0.4715 \\
\mathbf{0 . 4 8 1 5 ^ { \ddagger }}\end{array}$ & $\begin{array}{r}- \\
+2.1 \%\end{array}$ \\
\hline GOV2 & \multicolumn{4}{|c|}{ Title run } & \multicolumn{4}{|c|}{ Description run } & \multicolumn{4}{|c|}{ Hybrid run } \\
\hline Model & nDCG@2 & & $\mathrm{P} @ 20$ & & nDCG@2 & & $\mathrm{P} @ 20$ & & nDCG@2 & & $\mathrm{P} @ 20$ & \\
\hline BM25 & 4774 & - & & -3 & 0.4264 & - & & - & 0.4774 & - & & - \\
\hline BM25+RM3 & 0 & - & & - & & & & - & 0.4851 & - & & - \\
\hline $\begin{array}{c}\text { Zero-shot transfer } \\
\text { Vanilla }{ }_{\text {BERT }}\end{array}$ & & - & & - & & & & - & & - & & - \\
\hline & & & & & & & & & & +0.4 & & +0.5 \\
\hline $\begin{array}{l}\text { Multi-phase } \\
\text { Vanilla BERT } \\
\text { Sim-Pair BERT }\end{array}$ & $\begin{array}{l}0.5476 \\
\mathbf{0 . 5 7 4 3}^{\ddagger}\end{array}$ & $\begin{array}{c}- \\
+4.9 \%\end{array}$ & $\begin{array}{l}0.6302 \\
\mathbf{0 . 6 5 4 0}\end{array}$ & $\begin{array}{r}- \\
+3.8 \%\end{array}$ & $\begin{array}{l}0.5175 \\
\mathbf{0 . 5 4 0 6}^{\ddagger}\end{array}$ & $\begin{array}{r}- \\
+4.5 \%\end{array}$ & $\begin{array}{l}0.5772 \\
\mathbf{0 . 6 0 8 4}^{\ddagger}\end{array}$ & $\begin{array}{r}- \\
+5.4 \%\end{array}$ & $\begin{array}{l}0.5909 \\
\mathbf{0 . 5 9 9 8}\end{array}$ & $\begin{array}{l}- \\
+1.5 \%\end{array}$ & $\begin{array}{l}0.6604 \\
\mathbf{0 . 6 7 5 8}\end{array}$ & $\begin{array}{c}- \\
+2.3 \%\end{array}$ \\
\hline
\end{tabular}

models for 1 single epoch with a batch size of 32 training instances comprising a query and a passage. We use the Adam optimizer with a learning rate of $1 e^{-5}$ with warm up over the first $10 \%$ of the total training steps.

For queries in the left-out test fold, we set the rerank threshold to 100 as a trade-off between latency and effectiveness. We report the average performance across all test folds measured in terms of P@20 and nDCG@20 using pytrec_eval ${ }^{5}$ In this setting, our vanilla baseline corresponds to the pointwise trained BERT-MaxP model (Dai and Callan, 2019) initialized with monoBERT fine-tuned on MS MARCO instead of Google's BERT pretrained checkpoint without any prior fine-tuning on the text ranking task.

Table 10 reports the reranking effectiveness obtained using the multi-phase fine-tuning setting compared to the single-phase MS MARCO fine-tuning (zero-shot transfer setting) for both Robust04 and GOV2 collections. We report results obtained for reranking the top 100 documents retrieved by BM25 in both settings. Thanks to the additional in-domain fine-tuning on the target collection, the performance on both collections improves regardless of the topic field used. We notice in this setting that Sim-Pair BERT is able to achieve significant gains over the vanilla baseline on the GOV2 collection, confirming our hypothesis that the zero-shot domain transfer from MS MARCO was not sufficient for this collection.

Using the multi-phase fine-tuning setting BERT-based models are able to achieve better performance on descriptions compared to titles on Robust04 by $+7.5 \%$ and $+8.3 \%$ for the vanilla and Sim-Pair models respectively, despite the

\footnotetext{
5 https://pypi.org/project/pytrec-eval/
} 
Table 11: Reranking effectiveness with exact matching ablation at different phases of the multi-phase fine-tuning configuration of Sim-Pair BERT on Robust04 and GOV2 collections. MS refers to the MS MARCO fine-tuning phase and ID to the in-domain fine-tuning. Best performance is highlighted in bold. Significant improvements over the vanilla baseline with $p<0.05$ and $p<0.01$ after Bonferroni correction are indicated with $\dagger$ and $\ddagger$ respectively for the same setting. Significant inferiority with $p<0.05$ is marked with $*$. Change rate over the vanilla baseline are reported for each metric (\%).

\begin{tabular}{|c|c|c|c|c|c|c|c|c|c|c|c|c|c|}
\hline Robust04 & Marking & \multicolumn{4}{|c|}{ Title run } & \multicolumn{4}{|c|}{ Description run } & \multicolumn{4}{|c|}{ Hybrid run } \\
\hline Run & MS ID & nDCG@2 & 20 & $\mathrm{P} @ 20$ & & nDCG@2 & & $P @ 20$ & & nDCG@2 & 20 & $\mathrm{P} @ 20$ & \\
\hline Vanilla BERT & $-\quad-$ & 0.4995 & - & 0.4275 & & 0.5368 & & 0.4492 & - & 0.5546 & - & 0.4715 & \\
\hline Sim-Pair BERT & $\checkmark$ & 0.5058 & $+1.3 \%$ & 0.4371 & $+2.2 \%$ & $0.5479^{\dagger}$ & $+2.1 \%$ & $0.4574^{\dagger}$ & $+1.8 \%$ & $0.5701^{\ddagger}$ & $+2.8 \%$ & $0.4815^{7}$ & $+2.1 \%$ \\
\hline & & 0.4978 & $-0.3 \%$ & 0.4281 & $+0.1 \%$ & $0.5521^{\ddagger}$ & $\begin{array}{r}+2.9 \% \\
\end{array}$ & $0.4592^{\dagger}$ & $+2.2 \%$ & $0.5678^{\ddagger}$ & $\begin{array}{l}+2.4 \% \\
\end{array}$ & $0.4811^{\dagger}$ & $+2.0 \%$ \\
\hline B & $\checkmark$ & $0.4896^{*}$ & $-2.0 \%$ & 0.4239 & $-0.8 \%$ & 0.5344 & $-0.4 \%$ & 0.4504 & $+0.3 \%$ & 0.5500 & $-0.8 \%$ & 0.4665 & $-1.0 \%$ \\
\hline GOV2 & Marking & \multicolumn{4}{|c|}{ Title run } & \multicolumn{4}{|c|}{ Description run } & \multicolumn{4}{|c|}{ Hybrid run } \\
\hline Run & MS ID & nDCG@2 & & $\mathrm{P} @ 20$ & & nDCG@2 & & $P @ 20$ & & nDCG@2 & & $P @ 20$ & \\
\hline Vanilla BERT & $-\quad-$ & 0.5476 & - & 0.6302 & - & 0.5175 & - & 0.5772 & - & 0.5909 & - & 0.6604 & - \\
\hline Sim-Pair BERT & 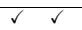 & $0.5743^{7}$ & $+4.9 \%$ & $0.6540^{\ddagger}$ & $+3.8 \%$ & $0.5406^{\ddagger}$ & $+4.5 \%$ & $0.6084^{\ddagger}$ & $+5.4 \%$ & 0.5998 & $+1.5 \%$ & 0.6758 & $+2.3 \%$ \\
\hline A & $\checkmark$ & $0.5665^{\dagger}$ & $+3.5 \%$ & 0.6430 & $+2.0 \%$ & $0.5509^{\ddagger}$ & $+6.5 \%$ & $0.6161^{\ddagger}$ & $+6.7 \%$ & 0.6027 & $\begin{array}{l}+2.0 \% \\
\end{array}$ & 0.6728 & $+1.9 \%$ \\
\hline B & $\checkmark$ & 0.5503 & $+0.5 \%$ & 0.6312 & $\begin{array}{l}+0.2 \% \\
\end{array}$ & 0.5218 & $+0.8 \%$ & 0.5785 & $+0.2 \%$ & 0.5761 & $-2.5 \%$ & 0.6517 & $-1.3 \%$ \\
\hline
\end{tabular}

lower retrieval effectiveness of BM25 on descriptions compared titles $(-4.3 \%)$. On the other hand, the difference in BM25 retrieval effectiveness between descriptions compared to titles is more important on GOV2, about $-11 \%$. The BERT-based rerankers reduce this gap to $-5.5 \%$ and $-5.9 \%$ for the vanilla and Sim-Pair models respectively but not enough to reverse the tendency. The endto-end effectiveness on this collection is thus higher on titles than descriptions as observed in previous state-of-the-art models such as BERT-MaxP(Dai and Callan, 2019) or PARADE(Li et al., 2020) (see results in section 5.5). Still, the hybrid pipeline outperforms both title and description runs on both collections. The reranking accuracy achieved by the hybrid runs are the highest reported results using a BERT-based model on both collections, at the time this article was written.

Phase-wise marking. Previous results of the Sim-Pair BERT model presented in Table 10 in the multi-phase setting are obtained using the exact match marking through out the two fine-tuning phases. While the first phase fine-tuning focuses on learning general notions of relevance from a large passage collection, the goal of adding in-domain fine-tuning is to learn directly from labeled data with the same distribution as the target task. It is important to determine on which of the two phases, the marking strategy is more beneficial and at which phase it can be omitted. To this aim, we conduct an ablation study on the Sim-Pair BerT model. Table 11 shows the results of the markingstrategy ablation on Robust04 and GOV2 collections using the different topic fields. With these results, we can now discuss our research question RQ3 At which phase the exact match marking is the most beneficial in a multi-phase fine-tuning configuration?

MS marking (labelled run $A$ in Table 11), uses exact match marking in the MS MARCO (MS) fine-tuning phase only then use the original data without further marking for the in-domain (ID) fine-tuning phase. We can see, in Table 
11. that using the marking strategy in the general fine-tuning phase is sufficient to outperform the vanilla baseline or at least perform similarly for Robust04 titles. In other words, initializing BERT with the weights learnt from marked inputs is better than those learnt from non-marked inputs. Ablating marking in the in-domain fine-tuning phase can even surpass the performance of the Sim-Pair BERT that uses marking across the two fine-tuning phases as observed for descriptions on both collections and the hybrid run on GOV2.

ID marking (labelled run B in Table 11), uses the marking strategy to augment the inputs during fine-tuning on the in-domain data while the BERT model was initialized with the weights learnt from non-marked MS MARCO inputs. The results of this first-phase marking ablation either has no substantial impact on the model's performance or leads to a degradation in performance. This behavior is predictable, since there is not enough in-domain data for BERT to learn useful representations of the marker tokens and their contribution to the relevance prediction.

Using a marking strategy during first general-purpose fine-tuning phase (MS marking) is already enough to outperform the vanilla baseline without requiring additional marking during the in-domain fine-tuning phase. At the end, the fine-tuned model using the Sim-Pair marking strategy on MS MARCO is able to use the relevance matching patterns learned using out-of-domain data, with explicit marking, for later phases even without the guidance of the explicit markers. Nevertheless, additional marking in the in-domain finetuning phase used in the classical Sim-Pair BERT approach is beneficial for title queries where it brings and additional gain of $+1.6 \%$ and $+1.4 \%$ over the MS marking only (run A) on Robust04 and GOV2, respectively.

\subsection{Impact of exact match marking on ELECTRA variant}

While BERT is the most famous and largely adopted pretrained language model, additional variants such as RoBERTa (Liu et al., 2019) or ELECTRA (Clark et al. 2020) were proposed in order to improve the model from different aspects. Recent state-of-the-art results reported on Robust04 and GOV2 collections were achieved using the ELECTRA model that appears to outperform BERT. ELECTRA (Clark et al. 2020) replaces the Masked Language Modeling (MLM) with a novel more sample-efficient pretraining task called replaced token detection. In this task, the model learns to distinguish real input tokens from plausible but synthetically generated replacements by a small "generator" model. This approach uses two components: the generator, a small two-layer BERT model that predicts masked tokens and the ELECTRA discriminator model that both require training. However, the new objective allows the model to learn from all input positions rather than only $15 \%$ of the positions in the MLM task.

In order to be confident in our approach, we investigate if exact match marking is beneficial for a BERT variant pretrained on a more robust task 
Table 12: Reranking effectiveness in the zero-shot transfer setting for the SimPair and vanilla models on Robust04 and GOV2 collections using both BERT and ELECTRA cores. Best performance is highlighted in bold. Significant improvements over the vanilla baseline with $p<0.05$ and $p<0.01$ are indicated with $\dagger$ and $\ddagger$ respectively for the same core. Significant inferiority with $p<0.05$ is marked with $*$. Change rate over the vanilla baseline for the same core type are reported for each metric (\%).

\begin{tabular}{|c|c|c|c|c|c|c|c|c|c|c|c|c|}
\hline Robust04 & \multicolumn{4}{|c|}{ Title run } & \multicolumn{4}{|c|}{ Description run } & \multicolumn{4}{|c|}{ Hybrid run } \\
\hline Model & $\mathrm{nDCG} @ 2$ & & $P @ 20$ & & nDCG@2 & & $\mathrm{P} @ 20$ & & nDCG@2 & & $P @ 20$ & \\
\hline BM25 & 0.4240 & - & 0.3631 & & 0.4058 & - & 0.3345 & - & 0.4240 & - & 0.3631 & - \\
\hline BM25+RM3 & 0.4407 & - & 0.3821 & - & 0.4255 & - & 0.3661 & - & 0.4407 & - & 0.3821 & - \\
\hline Vanilla BERT & 0.4652 & - & 0.4046 & & 0.4510 & - & 0.3851 & - & 0.4845 & - & 0.4147 & - \\
\hline Sim-Pair BERT & 0.4773 & $+2.6 \%$ & 0.4155 & $+2.7 \%$ & $0.4931^{\ddagger}$ & $+9.3 \%$ & $0.4169^{\ddagger}$ & $+8.3 \%$ & $0.5239^{\ddagger}$ & $+8.1 \%$ & $0.4446^{\ddagger}$ & $+7.2 \%$ \\
\hline Vanilla ELECTRA & 0.4416 & & 0.3833 & & 0.4482 & & 0.3831 & - & 0.4782 & - & 0.4141 & \\
\hline Sim-Pair ELECTRA & $0.4717^{\ddagger}$ & $+6.8 \%$ & 0.4124 & $+7.6 \%$ & 0.4597 & $+2.6 \%$ & 0.3886 & $+1.4 \%$ & $0.5043^{\ddagger}$ & $+5.5 \%$ & 0.4263 & $+2.9 \%$ \\
\hline GOV2 & \multicolumn{4}{|c|}{ Title run } & \multicolumn{4}{|c|}{ Description run } & \multicolumn{4}{|c|}{ Hybrid run } \\
\hline Model & nDCG@2 & & $P @ 20$ & & nDCG@2 & & $\mathrm{P} @ 20$ & & nDCG@2 & & $\mathrm{P} @ 20$ & \\
\hline BM25 & 0.4774 & - & 0.5362 & - & 0.4264 & - & 0.4705 & - & 0.4774 & - & 0.5362 & - \\
\hline BM25+RM3 & 0.4851 & - & 0.5634 & - & 0.4212 & - & 0.4966 & - & 0.4851 & - & 0.5634 & - \\
\hline Vanilla BERT & 0.4533 & - & 0.5272 & - & 0.4696 & - & 0.5248 & - & 0.4937 & - & 0.5611 & - \\
\hline Sim-Pair BERT & 0.4468 & $-1.4 \%$ & 0.5134 & $-2.6 \%$ & 0.4687 & $-0.2 \%$ & 0.5326 & $+1.5 \%$ & 0.4991 & $+1.1 \%$ & 0.5695 & $+1.5 \%$ \\
\hline Vanilla ELECTRA & 0.4668 & & 0.5332 & & 0.4986 & - & 0.5601 & - & 0.5147 & - & 0.5765 & - \\
\hline Sim-Pair ELECTRA & $0.4881^{\ddagger}$ & $+4.6 \%$ & $0.5577^{\ddagger}$ & $+4.6 \%$ & 0.5030 & $+0.9 \%$ & 0.5634 & $+0.6 \%$ & 0.5249 & $+2.0 \%$ & 0.5923 & $+2.7 \%$ \\
\hline
\end{tabular}

and study RQ4 Is exact match marking beneficial in alternative transformerbased models such as ELECTRA?

For our experiments, we use the base version of the ELECTRA model as the core of our model architecture illustrated in Figure 1 as a replacement of the BERT model. We use the same single-layer neural network that estimates a score $R(d, q)$ quantifying how relevant the candidate document $d$ is to the query $q$. We fine-tune the Vanilla electra and Sim-Pair Electra using the same hyper parameters used for their BERT counterparts.

\subsubsection{Zero-shot transfer setting}

We use the fine-tuned models on exclusively out-of-domain data, i.e MS MARCO passage dataset, and apply inference on the window-passages obtained by splitting each document using the same passage length of 150 words and a 75 words stride used in the BERT experiments. Table 12 shows the results obtained at cutoff 1,000 on both Robust04 and GOV2 collections. We recall the results of the Vanilla and Sim-Pair models with the BERT core for comparison.

Exact Match Marking on ELECTRA. Results indicate clearly that adding exact match marking is still beneficial for the ELECTRA variant. As for the BERT version, Sim-Pair ELECTRA is more effective on Robust04 with an average improvement rate of $+5 \%$ compared to only half, $+2.5 \%$, on GOV 2 . However, exact match marking has more notable impact on titles rather than descriptions, when the vanilla ELECTRA baseline prefers clearly description queries. 
Table 13: Reranking effectiveness in the multi-phase fine-tuning setting for the Sim-Pair and vanilla models on Robust04 and GOV2 collections using both BERT and ELECTRA cores. Best performance is highlighted in bold. Significant improvements over the vanilla baseline with $p<0.05$ and $p<0.01$ are indicated with $\dagger$ and $\ddagger$ respectively for the same core. Significant inferiority with $p<0.05$ is marked with $*$. Change rate over the vanilla baseline for the same core type are reported for each metric (\%).

\begin{tabular}{|c|c|c|c|c|c|c|c|c|c|c|c|c|}
\hline Robust04 & \multicolumn{4}{|c|}{ Title run } & \multicolumn{4}{|c|}{ Description run } & \multicolumn{4}{|c|}{ Hybrid run } \\
\hline Model & $\mathrm{nDCG} @ 2$ & & $P @ 20$ & & nDCG@2 & & $\mathrm{P} @ 20$ & & nDCG@2 & & $P @ 20$ & \\
\hline BM25 & 0.4240 & - & 0.3631 & & 0.4058 & - & 0.3345 & - & 0.4240 & - & 0.3631 & - \\
\hline $\mathrm{BM} 25+\mathrm{RM} 3$ & 0.4407 & - & 0.3821 & - & 0.4255 & - & 0.3661 & - & 0.4407 & - & 0.3821 & - \\
\hline Vanilla BERT & 0.4995 & - & 0.4275 & - & 0.5368 & - & 0.4492 & - & 0.5546 & - & 0.4715 & - \\
\hline Sim-Pair BERT & 0.5058 & $+1.3 \%$ & 0.4371 & $+2.2 \%$ & $0.5479^{\dagger}$ & $+2.1 \%$ & $0.4574^{\dagger}$ & $+1.8 \%$ & $0.5701^{\ddagger}$ & $+2.8 \%$ & $0.4815^{\ddagger}$ & $+2.1 \%$ \\
\hline Vanilla ELECTRA & 0.5375 & & 0.4560 & & 0.5676 & & 0.4663 & & 0.5901 & - & 0.4902 & \\
\hline Sim-Pair ELECTRA & 0.5380 & $+0.1 \%$ & 0.4564 & $+0.1 \%$ & 0.5686 & $+0.2 \%$ & 0.4705 & $+0.9 \%$ & 0.5927 & $+0.4 \%$ & 0.4942 & $+0.8 \%$ \\
\hline GOV2 & \multicolumn{4}{|c|}{ Title run } & \multicolumn{4}{|c|}{ Description run } & \multicolumn{4}{|c|}{ Hybrid run } \\
\hline Model & nDCG@2 & & $\mathrm{P} @ 20$ & & nDCG@2 & & $\mathrm{P} @ 20$ & & nDCG@2 & & $\mathrm{P} @ 20$ & \\
\hline BM25 & 0.4774 & - & 0.5362 & - & 0.4264 & - & 0.4705 & - & 0.4774 & - & 0.5362 & - \\
\hline BM25+RM3 & 0.4851 & - & 0.5634 & - & 0.4212 & - & 0.4966 & - & 0.4851 & - & 0.5634 & - \\
\hline Vanilla BERT & 0.5476 & 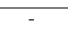 & 0.6302 & & 0.5175 & - & 0.5772 & - & 0.5909 & - & 0.6604 & - \\
\hline Sim-Pair BERT & $0.5743^{\ddagger}$ & $+4.9 \%$ & $0.6540^{\ddagger}$ & $+3.8 \%$ & $0.5406^{\ddagger}$ & $+4.5 \%$ & $0.6084^{\ddagger}$ & $+5.4 \%$ & 0.5998 & $+1.5 \%$ & 0.6758 & $+2.3 \%$ \\
\hline Vanilla ELECTRA & 0.5784 & - & 0.6621 & & 0.5629 & - & 0.6279 & - & 0.6149 & - & 0.6862 & - \\
\hline Sim-Pair ELECTRA & 0.5868 & $+1.5 \%$ & 0.6661 & $+0.6 \%$ & 0.5552 & $-1.4 \%$ & 0.6225 & $-0.9 \%$ & 0.6133 & $-0.3 \%$ & 0.6926 & $+0.9 \%$ \\
\hline
\end{tabular}

ELECTRA vs. BERT core. The Sim-Pair ELECTRA variant achieves better performance than its BERT counterpart regardless of the topic field on the GOV2 collection. In contrast, using the BERT core is more effective on Robust04 on both titles, descriptions and the hybrid pipeline. The same tendency can be observed for the vanilla baseline with smaller margins.

\subsubsection{Multi-phase fine-tuning}

Table 13 shows the results obtained using the multi-phase fine-tuning on both MS MARCO passage dataset and in-domain labeled data, described in section 5.3 for BERT. The ELECTRA-based models outperform the BERT-based models on both collections regardless of the topic field used indicating that ELECTRA is a more effective core PLM than BERT in a mlti-phase finetuning setting. However, adding exact match marking has no significant impact in this setting. Sim-Pair ELECTRA performs slightly better than the vanilla ELECTRA baseline on the Robust04 collection across title, description and hybrid runs. On the other hand, exact match marking leads to better ranking accuracy on GOV2 titles, but provokes a slight degradation in performance when the description field is used for reranking (description and hybrid runs).

Summary. Exact match marking is indeed beneficial for the ELECTRA model, especially in a zero-shot transfer setting where no labeled data is available in the target domain. Sim-Pair ELECTRA is able to achieve significant gains on titles, where Sim-Pair BERT is less effective. However, for description and hybrid runs that use descriptions for reranking, exact match marking appears to have more substantial impact when using a BERT core. Finally, we can 
Table 14: Characteristics of state-of-the-art models.

\begin{tabular}{lllll}
\hline Model & Ranking formulation & Document splitting & Score estimation & PLM \\
\hline Birch & Relevance classification & Sentences & $\begin{array}{l}\text { Top-n scoring sentences } \\
\text { combined with BM25 scores }\end{array}$ & BERT Large \\
\hline BERT-MaxP & Relevance classification & Window passages & Top scoring passage & BERT $_{\text {Base }}$ \\
\hline Parade & Relevance classification & Window passages & Passage representation aggregation & BERT $_{\text {Base }}-$ ELECTRA $_{\text {Base }}$ \\
\hline T5 & Seq2Seq & Window passages & Top scoring passage & T5 \\
\hline
\end{tabular}

say that, in most cases, the ELECTRA-based versions of our models are more effective compared to their BERT counterparts.

\subsection{Comparison with state-of-the-art models}

In this section we try to situate our approach with regard to what has already been proposed for document ranking. We consider the following models that represent the state of the art:

Birch (MS) and Birch (MS-MB). Aggregates document-level scores provided by BM25 with the sentence-level evidence from BERT to rank the documents (Akkalyoncu Yilmaz et al., 2019). The notation in parentheses indicate the fine-tuning dataset(s): Ms for MS MARCO and MS-MB refers to the model fine-tuned first on MS MARCO and then further fine-tuned on Microblog (MB) data. We use the results reported by Li et al. (2020) that uses BM25 instead of BM25+RM3 as the first-stage retriever.

BERT-MaxP (MS). Uses the best-scoring passage as a proxy for the document relevance score (Dai and Callan, 2019). We report the results obtained with the reimplementation by $\mathrm{Li}$ et al. (2020) where the results are improved using a BERT model fine-tuned on MS MARCO rather than Bing search log.

Parade. Aggregates passages representations rather than passage scores to determine the document relevance score (Li et al., 2020). We report results obtained using both BERT and ELECTRA variant.

T5. Adapts the T5 pretrained sequence-to-sequence model for document ranking (Nogueira et al., 2020). The model is fine-tuned to produce the tokens "true" or "false" depending on whether the document is relevant or not to the query. The T5 with $3 B$ parameters detains the state-of-the-art across many ad hoc benchmarks like Robust04.

Table 14 summarizes some of the important characteristics of these baselines: the formulation of the ranking problem, document splitting, final document score estimation and the PLM used. In our approach, we adopted the straightforward formulation of ranking as a classification problem of the documents as relevant or not w.r.t a given query. We also focused on the widely adopted score aggregation method to determine the final document-level score from the individual passage scores. Considering these aspects, the Birch and BERT-MaxP models have the closest configuration for a fair comparison. 
Table 15: Reranking effectiveness of the Sim-Pair BERT with interpolating BM25 scores vs. Birch (MS) baseline on both Robust04 and GOV2 collections.

\begin{tabular}{l|llll|llll}
\hline Robust04 & \multicolumn{4}{|c|}{ Title run } & \multicolumn{4}{c}{ Description run } \\
\hline Model & nDCG@20 & P@20 & nDCG@20 & P@20 \\
\hline BM25 & 0.4240 & - & 0.3631 & - & 0.4058 & - & 0.3345 & - \\
\hline Birch (MS) & 0.4227 & - & 0.3616 & - & 0.4053 & - & 0.3341 & - \\
Sim-Pair BERT + BM25 & $\mathbf{0 . 4 9 4 7}$ & $+17 . \%$ & $\mathbf{0 . 4 2 6 5}$ & $+18 . \%$ & $\mathbf{0 . 5 0 9 8}$ & $+26 . \%$ & $\mathbf{0 . 4 2 7 9}$ & $+28 . \%$ \\
\hline \hline GOV2 & \multicolumn{3}{|c|}{ Title run } & \multicolumn{4}{c}{ Description run } \\
\hline Model & nDCG@20 & P@20 & \multicolumn{4}{|c|}{ nDCG@20 } & P@20 \\
\hline BM25 & 0.4774 & - & 0.5362 & - & 0.4264 & - & 0.4705 & - \\
\hline Birch (MS) & 0.4722 & - & 0.5352 & - & 0.4260 & - & 0.4701 & - \\
Sim-Pair BERT + BM25 & $\mathbf{0 . 5 3 2 7}$ & $+13 . \%$ & $\mathbf{0 . 6 0 0 0}$ & $+12 . \%$ & $\mathbf{0 . 5 2 3 5}$ & $+23 . . \%$ & $\mathbf{0 . 5 8 9 3}$ & $+25 . \%$ \\
\hline
\end{tabular}

\subsubsection{Comparison in the same experimental design.}

In order to fairly compare a novel approach with previously proposed ones, it is important to conduct the evaluation in the same experimental conditions. Here, we try to reproduce as much of the original settings used to produce the results of the Birch and BERT-maxP baselines, respectively.

Birch (MS). This baseline is fine-tuned exclusively on MS MARCO passages, therefore we use our Sim-Pair BERT + BM25 model equally fine-tuned on MS MARCO passages and augmented with BM25 scores interpolation following the same Equation 4 used in Birch (Akkalyoncu Yilmaz et al., 2019). All Robust04 and GOV2 topics and relevance judgements are used as a held-out test set.

Table 15 shows the results of our Sim-Pair BERT + BM25 model compared to the Birch (MS) baseline. The results clearly indicate that our model outperforms Birch (MS). Since our model already outperforms the baseline with a BERT Base version, it unnecessary to conduct the same experiment with a BERT Large whose computational cost is, unfortunately, beyond our hardware limitation 6 .

BERT-MaxP (MS). The configuration of this baseline is the same we used in the multi-phase fine-tuning setting. We compare the results of Sim-Pair BERT fine-tuned first on MS MARCO and then further fine-tuned on the target task obtained with a 5 -fold cross validation with BERT-MaxP (MS) in Table 16. We report the results when using the exact match marking during fine-tuning on MS MARCO passages only [MS], and the results with the full marking on both MS MARCO and in-domain data [MS+ID]. Our approach outperforms clearly the BERT-maxP baseline on titles, and performs slightly better on descriptions. It is important to notice that the BERT-MaxP results reported

\footnotetext{
6 We tried fine-tuning a Large version of BERT on Google Colab free TPUs, but it appears that the TF2 implementation of BERT from HuggingFace's Transformers library requires more than the free allocated resources on Google Colab to fit the hyper-parameters adopted in literature (Nogueira and Cho, 2019)
} 
Table 16: Reranking effectiveness of the Sim-Pair BERT with multi-phase finetuning vs. BERT-MaxP (MS) baseline on both Robust04 and GOV2 collections. [MS] indicates that the run uses MS marking: exact match marking is only used during fine-tuning on MS MARCO and ablated in the in-domain fine-tuning phase.

\begin{tabular}{|c|c|c|c|c|c|c|c|c|}
\hline Robust04 & \multicolumn{4}{|c|}{ Title run } & \multicolumn{4}{|c|}{ Description run } \\
\hline Model & nDCG@ & & $\mathrm{P} @ 20$ & & nDCG@ & & $\mathrm{P} @ 20$ & \\
\hline BM25 & 0.4240 & - & 0.3631 & - & 0.4058 & - & 0.3345 & - \\
\hline BERT-MaxP (MS) & 0.4931 & - & 0.4277 & - & 0.5453 & - & 0.4522 & - \\
\hline Sim-Pair BERT & 0.5058 & $+2.6 \%$ & 0.4371 & $+2.2 \%$ & 0.5479 & $+0.5 \%$ & 0.4574 & $+1.1 \%$ \\
\hline Sim-Pair BERT [MS] & 0.4978 & $+1.0 \%$ & 0.4281 & $+0.1 \%$ & 0.5521 & $+1.2 \%$ & 0.4592 & $+1.5 \%$ \\
\hline GOV2 & \multicolumn{4}{|c|}{ Title run } & \multicolumn{4}{|c|}{ Description run } \\
\hline Model & nDCG@ & & $\mathrm{P} @ 20$ & & nDCG@ & & $\mathrm{P} @ 20$ & \\
\hline BM25 & 0.4774 & - & 0.5362 & - & 0.4264 & - & 0.4705 & - \\
\hline BERT-MaxP (MS) & 0.5600 & - & 0.6352 & - & 0.5506 & - & 0.6087 & - \\
\hline Sim-Pair BERT & 0.5743 & $+2.6 \%$ & 0.6540 & $+3.0 \%$ & 0.5406 & $-1.8 \%$ & 0.6084 & $-0.0 \%$ \\
\hline Sim-Pair BERT [MS] & 0.5665 & $+1.2 \%$ & 0.6430 & $+1.2 \%$ & 0.5509 & $+0.1 \%$ & 0.6161 & $+1.2 \%$ \\
\hline
\end{tabular}

Table 17: Reranking effectiveness on Robust04 and GOV2 of our best runs vs. the best baseline runs. The change rate (\%) of our best run, Sim-Pair ELECTRA, over each baseline is indicated for both metrics if available. We use the multi-phase fine-tuning for our runs, the same multi-phase fine-tuning is adapted in Parade and BERT-maxP baselines.

\begin{tabular}{|c|c|c|c|c|c|c|c|c|c|c|}
\hline \multirow{2}{*}{$\begin{array}{l}\text { Hybrid runs } \\
\text { Model }\end{array}$} & \multicolumn{5}{|c|}{ Robust04 } & \multicolumn{5}{|c|}{ GOV2 } \\
\hline & \multicolumn{2}{|c|}{ nDCG@20 } & \multicolumn{2}{|c|}{$\mathrm{P} @ 20$} & \multirow{2}{*}{$\begin{array}{c}\text { Field } \\
\text { Title }\end{array}$} & \multicolumn{2}{|c|}{ nDCG@20 } & \multicolumn{2}{|l|}{$\mathrm{P} @ 20$} & Field \\
\hline Birch (MS-MB) & 0.5137 & $+15 . \%$ & 0.4404 & $+12 . \%$ & & 0.5608 & $+9.4 \%$ & 0.6409 & $8.1 \%$ & Title \\
\hline BERT-MaxP (MS) & 0.5453 & $+8.7 \%$ & 0.4522 & $+9.3 \%$ & Desc & 0.5600 & $+9.5 \%$ & 0.6356 & $+9.0 \%$ & Title \\
\hline Parade & 0.5605 & $+5.7 \%$ & 0.4661 & $+6.0 \%$ & Desc & 0.5750 & $+6.7 \%$ & 0.6530 & $+6.1 \%$ & Title \\
\hline Parade (ELECTRA) & 0.5713 & $+3.7 \%$ & 0.4717 & $+4.8 \%$ & Desc & 0.5851 & $+4.8 \%$ & 0.6678 & $+3.7 \%$ & Title \\
\hline T5-base & 0.5298 & $+12 . \%$ & - & - & Hybrid & - & - & - & - & - \\
\hline T5-large & 0.5345 & $+11 . \%$ & - & - & Hybrid & - & - & - & - & - \\
\hline T5-3B & 0.6091 & $-2.7 \%$ & - & - & Hybrid & - & - & - & - & - \\
\hline Sim-Pair BERT & 0.5701 & - & 0.4815 & - & Hybrid & 0.5998 & - & 0.6758 & - & Hybrid \\
\hline Sim-Pair ELECTRA & 0.5927 & - & 0.4942 & - & Hybrid & 0.6133 & - & 0.6926 & - & Hybrid \\
\hline
\end{tabular}

by Li et al. (2020) are better than our vanilla BERT baseline in the multiphase fine-tuning setting, especially on GOV2. This difference indicates that our implementations are different and suspect that we did not use the same loss function: pointwise vs. pairwise.

\subsubsection{Comparison with different experimental designs}

Each approach has the optimal experimental conditions that lead to the best ranking accuracy possible, and these optimal conditions are hardly the same for the different models we want to compare. Independently of the experimental framework employed to obtain the results, Table 17 compares our best runs with both BERT and ELECTRA cores obtained in the multi-phase fine-tuning setting, and the best baseline runs. Results obtained using the Sim-Pair BERT model outperform all the BERT-based models that represent the state of the 
art and achieves better performance than $\mathrm{T} 5$ for both base and large versions on robust04. The Sim-Pair ELECTRA variant achieves comparable performance with the T5-3B model while using only $110 M$ parameters and outperforms the Parade (ELECTRA) model on both Robust04 and GOV2 collections by a margin of $3 \%$ to more than $4 \%$. The T5 baseline is by far the strongest baseline, it is important to note that it uses a zero-shot transfer setting without the need for in-domain fine-tuning as opposed to BERT-MaxP, Parade and our own models, however, its large size make it unpractical compared to a $\mathrm{BERT}_{\text {Base }}$ or ELECTRA Base.

\section{Discussion and Future Work}

Our research is related to effectively harnessing the exact matching signals from the query-document pairs to enhance document ranking with pretrained language models (PLMs) exemplified by BERT. We have shown through the empirical experiments reported in this paper that PLMs such as BERT can benefit from explicit exact match cues conveyed via marker tokens to be more effective for ad hoc ranking.

BERT as the most famous PLM, was successfully applied to text ranking as well as a wide range of other tasks without requiring any specialized neural architectural components to capture different relevance signals as opposed to pre-BERT neural ranking models. Previous work by Qiao et al. (2019) study the behaviour of BERT for ranking and find that it is able to capture semantic matching signals between paraphrase tokens. However, research from the preBERT era have proven that, in addition to semantic matching, exact matching is still an important cue for neural ranking models (Guo et al., 2016: Mitra et al. 2017). Guo at al. (Guo et al. 2016) argue that "exact matching of terms in documents with those in queries is still the most important signal in ad-hoc retrieval due to the indexing and search paradigm in modern search engines". This is why, Boualili et al. (2020) suggest to emphasize the exact match signals for BERT using a marking technique that does not involve redesigning the model's architecture, which will cost the immense benefits of self-supervised pretraining.

In this paper we extend Boualili et al. (2020) and study four research questions that aim to investigate the effectiveness of our newly proposed marking strategies for ad hoc document ranking.

First, we investigated the benefits of exact match marking for a BERTbased model in a zero-shot transfer setting. The results of the experiment showed that combining a simple soft marker with a pair marking strategy (SimPair) is the most simple yet effective marking strategy. Moreover, this exact match marking approach has a higher effectiveness on the description field of the topic compared to the title field. This preference for well-written natural language questions is in line with BERT's preference for descriptions revealed by Dai and Callan (2019). On the other hand, we follow a retrieve-then-rerank architecture where the retriever is a bag-of-words model that prefers short 
key word queries while the reranker is a BERT-based model that prefers long natural language questions (Dai and Callan, 2019, Nogueira et al. 2020). In order to get the best of the two stages, we propose a hybrid pipeline where titles are used during the retrieving stage and then replaced by descriptions in the reranking stage which leads to substantial gains in performance.

Second, we find exact term matching scores from traditional bag-of-words models like BM25 are still beneficial for BERT-based document ranking. Indeed, combining document-level scores from BM25 with passage-evidence from out BERT-based models with a simple linear interpolation leads to substantial gains in performance. The document-level scores from initial BM25 retrieval based on traditional IR cues (TF, IDF) provide additional relevance signals that complete the passage-level scores from BERT-based models. Furthermore, using exact match marking appears to better take advantage of the combination with BM25 scores to achieve better performance than the vanilla model.

Third, when adding in-domain fine-tuning on top of the first generalpurpose fine-tuning phase on out-of-domain data, we demonstrated through an ablation study that using exact match marking in the general-purpose finetuning phase on large out-of-domain data is enough to achieve substantial leaps in performance especially on descriptions. We publish our fine-tuned checkpoints on MS MARCO so it can be accessible to the community as a more effective alternative to a vanilla checkpoint.

Fourth, we study the contribution of our exact match marking strategy on a BERT variant, ELECTRA, that has been recently used in state of the art models such as Parade (Li et al., 2020). Experiments showed that exact match marking is indeed beneficial for ELECTRA, especially in the zero-shot transfer setting where no in-domain annotated data is used for training. In addition, the ELECTRA-based models were able to outperform their BERT counterparts in most cases.

Finally, we compared our best runs using both BERT and ELECTRA to a wide range of transformer-based ranking models that represent the state of the art at the time this article was written. The comparative evaluation showed that our exact match marking approach combined with the hybrid pipeline, that uses titles for BM25 retrieval and descriptions for BERT reranking, achieves near state-of-the-art results on Robust04 compared to the strong and larger T5-3B baseline, and outperforms previously proposed models on GOV2.

Our approach was empirically proven to be effective on standard ad hoc benchamrks, however in terms of explicability, there is still a lot of analysis that need to be done in order to understand how exactly the marking conveys the exact match signals to BERT and how are they integrated in the relevance prediction process. To this day, only so little is understood about the inner workings of BERT and PLMs in general regardless of all the efforts put into studying their behaviors. Previous research attempted to reveal insights about how BERT "works" in the limited context of passage retrieval, but studies lack when it comes to long documents ranking. Aside from the explicability limitation, our approach is rather simple and considers all query terms to be 
of equal importance when, in reality, they hardly have the same importance in the query especially in long descriptions.

For future work, we plan to develop diagnostic tests in attempt to shed light on the contribution of the exact match marking to the inner workings of BERT. Once the intervention of the markers determined, their representations can be leveraged for relevance classification in addition or instead of the current standard [CLS]. Identifying the subset of queries that are most likely to be improved by adding explicit exact match cues can be used to choose whether to use marking or not. Finally, our approach could be further improved by integrating the query term importance.

\section{Conclusion}

Pretrained language models perform well on an impressively wide range of tasks. They were proven to excel at semantic matching, nevertheless exact matching is essential for relevance matching. In the light of this fact, we proposed to use marker tokens to convey exact match cues from the textual input that yield strong performance while maintaining the same architecture i.e number of parameters. We showed through empirical experiments that using a simple marker combined with a pair marking level is the most simple strategy that yields the best effectiveness. We show that applying this marking strategy in a hybrid retrieve-than-rerank pipeline that uses short key word queries for the first bag-of-words retriever and then adopts long natural language queries for reranking with PLMs like BERT and ELECTRA produce competitive effectiveness compared to state-of-the-art models. We published our fine-tuned checkpoints on marked data on the HuggingFace model hub so it can be easily used by the community via the famous "transformers" library without changes to their setups while benefiting from the improvements brought by exact match marking and build upon them.

\section{References}

Akkalyoncu Yilmaz Z, Yang W, Zhang H, Lin J (2019) Cross-domain modeling of sentence-level evidence for document retrieval. In: Proc. of the 2019 EMNLP-IJCNLP Conf., ACL, Hong Kong, China, pp 3488-3494

Boualili L, Moreno JG, Boughanem M (2020) Markedbert: Integrating traditional ir cues in pre-trained language models for passage retrieval. Association for Computing Machinery, New York, NY, USA, SIGIR '20, p 1977-1980

Câmara A, Hauff C (2020) Diagnosing bert with retrieval heuristics. In: Jose JM, Yilmaz E, Magalhães J, Castells P, Ferro N, Silva MJ, Martins F (eds) Advances in Information Retrieval, Springer International Publishing, Cham, pp 605-618 
Clark K, Luong MT, Le QV, Manning CD (2020) Electra: Pre-training text encoders as discriminators rather than generators. In: International Conference on Learning Representations

Craswell N, Mitra B, Yilmaz E, Campos D, Voorhees EM (2020) Overview of the TREC 2019 deep learning track. CoRR abs/2003.07820

Dai Z, Callan J (2019) Deeper text understanding for ir with contextual neural language modeling. In: Proceedings of the 42nd International ACM SIGIR Conference on Research and Development in Information Retrieval, pp 985988

Dai Z, Xiong C, Callan J, Liu Z (2018) Convolutional neural networks for softmatching n-grams in ad-hoc search. Association for Computing Machinery, New York, NY, USA, WSDM '18, p 126-134

Devlin J, Chang M, Lee K, Toutanova K (2019) BERT: Pre-training of deep bidirectional transformers for language understanding. In: Proc. of the 2019 NAACL-HLT Conf., Volume 1, ACL, pp 4171-4186

Guo J, Fan Y, Ai Q, Croft W (2016) A deep relevance matching model for adhoc retrieval. In: Proceedings of the 25th ACM International on Conference on Information and Knowledge Management, pp 55-64

Hofstätter S, Zamani H, Mitra B, Craswell N, Hanbury A (2020) Local selfattention over long text for efficient document retrieval. Association for Computing Machinery, New York, NY, USA, SIGIR '20, p 2021-2024

Khattab O, Zaharia M (2020) Colbert: Efficient and effective passage search via contextualized late interaction over bert. Association for Computing Machinery, New York, NY, USA, SIGIR '20, p 39-48

Kingma DP, Ba J (2015) Adam: A method for stochastic optimization. In: Bengio Y, LeCun Y (eds) 3rd International Conference on Learning Representations, ICLR 2015, San Diego, CA, USA, May 7-9, 2015, Conference Track Proceedings

Lavrenko V, Croft WB (2001) Relevance based language models. In: Proceedings of the 24th Annual International ACM SIGIR Conference on Research and Development in Information Retrieval, Association for Computing Machinery, New York, NY, USA, SIGIR '01, p 120-127

Li C, Yates A, MacAvaney S, He B, Sun Y (2020) PARADE: passage representation aggregation for document reranking. CoRR abs/2008.09093

Li H (2011) Learning to Rank for Information Retrieval and Natural Language Processing. Morgan \& Claypool Publishers

Liu TY (2009) Learning to rank for information retrieval. Found Trends Inf Retr 3(3):225-331

Liu Y, Ott M, Goyal N, Du J, Joshi M, Chen D, Levy O, Lewis M, Zettlemoyer L, Stoyanov V (2019) Roberta: A robustly optimized BERT pretraining approach. CoRR abs/1907.11692

Luan Y, Eisenstein J, Toutanova K, Collins M (2020) Sparse, dense, and attentional representations for text retrieval. CoRR abs/2005.00181

MacAvaney S, Yates A, Cohan A, Goharian N (2019) Cedr: Contextualized embeddings for document ranking. In: Proceedings of the 42nd International ACM SIGIR Conference on Research and Development in Information Re- 
trieval, pp 1101-1104

MacAvaney S, Feldman S, Goharian N, Downey D, Cohan A (2020a) ABNIRML: analyzing the behavior of neural IR models

MacAvaney S, Nardini FM, Perego R, Tonellotto N, Goharian N, Frieder O (2020b) Expansion via Prediction of Importance with Contextualization, Association for Computing Machinery, New York, NY, USA, p 1573-1576

Mitra B, Diaz F, Craswell N (2017) Learning to match using local and distributed representations of text for web search. In: Proceedings of the 26th International Conference on World Wide Web, pp 1291-1299

Mitra B, Craswell N, others (2018) An introduction to neural information retrieval. Foundations and Trends(R) in Information Retrieval 13(1):1-126

Nguyen T, Rosenberg M, Song X, Gao J, Tiwary S, Majumder R, Deng L (2016) MS MARCO: A human generated machine reading comprehension dataset. CoRR abs/1611.09268

Nogueira R, Cho K (2019) Passage re-ranking with BERT. CoRR

Nogueira R, Yang W, Cho K, Lin J (2019) Multi-stage document ranking with BERT. CoRR abs/1910.14424

Nogueira R, Jiang Z, Pradeep R, Lin J (2020) Document ranking with a pretrained sequence-to-sequence model. In: Findings of the Association for Computational Linguistics: EMNLP 2020, Association for Computational Linguistics, Online, pp 708-718

Onal KD, Zhang Y, Altingovde IS, Rahman MM, Karagoz P, Braylan A, Dang B, Chang HL, Kim H, Mcnamara Q, Angert A, Banner E, Khetan V, Mcdonnell T, Nguyen AT, Xu D, Wallace BC, Rijke M, Lease M (2018) Neural information retrieval: At the end of the early years. Information Retrieval Journal 21(2-3):111-182

Padaki R, Dai Z, Callan J (2020) Rethinking query expansion for bert reranking. In: Jose JM, Yilmaz E, Magalhães J, Castells P, Ferro N, Silva MJ, Martins F (eds) Advances in Information Retrieval, Springer International Publishing, Cham, pp 297-304

Padigela H, Zamani H, Croft WB (2019) Investigating the successes and failures of BERT for passage re-ranking. CoRR abs/1905.01758

Pradeep R, Nogueira R, Lin J (2021) The expando-mono-duo design pattern for text ranking with pretrained sequence-to-sequence models. CoRR abs/2101.05667

Qiao Y, Xiong C, Liu Z, Liu Z (2019) Understanding the behaviors of BERT in ranking

Raffel C, Shazeer N, Roberts A, Lee K, Narang S, Matena M, Zhou Y, Li W, Liu PJ (2020) Exploring the limits of transfer learning with a unified textto-text transformer. Journal of Machine Learning Research 21(140):1-67

Rennings D, Moraes F, Hauff C (2019) An axiomatic approach to diagnosing neural ir models. In: Azzopardi L, Stein B, Fuhr N, Mayr P, Hauff C, Hiemstra D (eds) Advances in Information Retrieval, Springer International Publishing, Cham, pp 489-503

Vaswani A, Shazeer N, Parmar N, Uszkoreit J, Jones L, Gomez AN, Kaiser Ł, Polosukhin I (2017) Attention is all you need. In: Advances in neural 
information processing systems, pp 5998-6008

Voorhees E, Alam T, Bedrick S, Demner-Fushman D, Hersh WR, Lo K, Roberts K, Soboroff I, Wang LL (2021) Trec-covid: Constructing a pandemic information retrieval test collection. SIGIR Forum 54(1), DOI $10.1145 / 3451964.3451965$

Wolf T, Debut L, Sanh V, Chaumond J, Delangue C, Moi A, Cistac P, Rault T, Louf R, Funtowicz M, Davison J, Shleifer S, von Platen P, Ma C, Jernite Y, Plu J, Xu C, Le Scao T, Gugger S, Drame M, Lhoest Q, Rush A (2020) Transformers: State-of-the-art natural language processing. In: Proceedings of the 2020 Conference on Empirical Methods in Natural Language Processing: System Demonstrations, Association for Computational Linguistics, Online, pp 38-45

Xiong C, Dai Z, Callan J, Liu Z, Power R (2017) End-to-end neural ad-hoc ranking with kernel pooling. In: Proc. of the 40th SIGIR Conf., pp 55-64

Yang P, Fang H, Lin J (2017) Anserini: Enabling the use of lucene for information retrieval research. In: Proceedings of the 40th International ACM SIGIR Conference on Research and Development in Information Retrieval, pp 1253-1256

Yang W, Lu K, Yang P, Lin J (2019a) Critically examining the "neural hype" weak baselines and the additivity of effectiveness gains from neural ranking models. In: Proceedings of the 42nd International ACM SIGIR Conference on Research and Development in Information Retrieval, pp 1129-1132

Yang W, Zhang H, Lin J (2019b) Simple applications of BERT for ad hoc document retrieval. CoRR abs/1903.10972

Yates A, Nogueira R, Lin J (2021) Pretrained transformers for text ranking: Bert and beyond. Association for Computing Machinery, New York, NY, USA, WSDM '21, p 1154-1156 
Table 18: Reranking effectiveness in the zero-shot transfer setting of all our models on Robust04 and GOV2 collections. Best results, for each cutoff, are highlighted in bold. Significant improvements over the Vanilla baseline with $p<0.05$ and $p<0.01$ are indicated with $\dagger$ and $\ddagger$ respectively, for the same cutoff. Significant inferiority with $p<0.05$ is marked with $*$. For each measure, the improvement rate over the Vanilla baseline is given (\%).

\begin{tabular}{|c|c|c|c|c|c|c|c|c|c|c|c|c|}
\hline Robust04 & & Title & run & & & Descrip & tion run & & & Hybri & id run & \\
\hline Model & nDCG@ & 20 & $P @ 20$ & & nDCG@2 & 20 & $P @ 20$ & & nDCG@2 & 20 & $\mathrm{P} @ 20$ & \\
\hline BM25 & 0.4240 & - & 0.3631 & - & 0.4058 & - & 0.3345 & - & 0.4240 & - & 0.3631 & - \\
\hline BM25+RM3 & 0.4407 & - & 0.3821 & - & 0.4255 & - & 0.3661 & - & 0.4407 & & 0.3821 & - \\
\hline Top-100 & & & & & & & & & & & & \\
\hline Vanilla & 1764 & - & 0.4096 & - & .4611 & - & 0.3867 & - & 0.4989 & - & 0.4245 & - \\
\hline & 1678 & $-1.8 \%$ & 0.4042 & $-1.3 \%$ & & $+0.1 \%$ & 3865 & $-0.1 \%$ & 4912 & $-1.5 \%$ & 0.4129 & $-2.7 \%$ \\
\hline & 4763 & $-0.0 \%$ & 0.4129 & +0.8 & $23^{\ddagger}$ & $+6.8 \%$ & $0.4084^{\ddagger}$ & +5 . & $0.5273^{\ddagger}$ & $+5.7 \%$ & $0.4434^{\ddagger}$ & $+4.5 \%$ \\
\hline & 4781 & $+0.4 \%$ & 78 & & & & & & $0.5205^{\ddagger}$ & $+4.3 \%$ & $0.4294^{\ddagger}$ & $+1.2 \%$ \\
\hline Pre-Pa & 700 & $-1.3 \%$ & 0.40 & & & +4 & & & $0.5132^{\ddagger}$ & $+2.9 \%$ & $0.4410^{\ddagger}$ & $+3.9 \%$ \\
\hline Top-1000 & & & & & & & & & & & & \\
\hline & & - & 0.4046 & - & 4510 & & 0.3851 & - & 0.4845 & - & 0.4147 & - \\
\hline & & $-4.4 \%$ & $3831^{*}$ & $-5.3 \%$ & & & & $-8.9 \%$ & $4476^{*}$ & $-7.6 \%$ & $3817^{*}$ & $-7.9 \%$ \\
\hline & 4773 & $+2.6 \%$ & 0.4155 & $+2.7 \%$ & $31^{\ddagger}$ & $+9.3 \%$ & $0.4169^{\ddagger}$ & $+8.3 \%$ & $0.5239^{\ddagger}$ & $+8.1 \%$ & $0.4446^{\ddagger}$ & $+7.2 \%$ \\
\hline & & $+2.5 \%$ & 084 & +0 & & +6 & & & & $+3.9 \%$ & & $+2.1 \%$ \\
\hline Pre-Pair ${ }_{\text {BERT }}$ & 0.4654 & $+0.0 \%$ & 0.4024 & $-0.5 \%$ & $0.4795^{\ddagger}$ & $+6.3 \%$ & $0.4034^{\ddagger}$ & $+4.8 \%$ & $0.5086^{\ddagger}$ & $+5.0 \%$ & $0.4319^{\ddagger}$ & $+4.1 \%$ \\
\hline GOV2 & & Title & run & & & Descrip & tion run & & & Hybri & id run & \\
\hline Model & $\overline{\mathrm{DCG} @}$ & & $P @ 20$ & & nDCG@2 & & $P @ 20$ & & nDCG@2 & & $\mathrm{P} @ 20$ & \\
\hline BM: & & 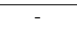 & 0.5362 & - & & & & & 0.4774 & - & 0.5362 & - \\
\hline & & & & & & & & . & 51 & & & - \\
\hline Top-100 & & & & & & & & & & & & \\
\hline & & - & 0.5916 & - & & - & & - & 0.55 & - & & - \\
\hline & & $+0.9 \%$ & & $+0.3 \%$ & & $-0.9 \%$ & & $-0.1 \%$ & & $-0.2 \%$ & & $+0.7 \%$ \\
\hline & & $+1.6 \%$ & & & & $-0.5 \%$ & & & & $+0.4 \%$ & & $+0.5 \%$ \\
\hline & & -0 & & & & & & & & $+1.1 \%$ & & $+0.2 \%$ \\
\hline & & & 0 & & & & & & .5559 & $+0.9 \%$ & 0.6332 & $+0.3 \%$ \\
\hline Top-1 & & & & & & & & & & & & \\
\hline & & & & - & & - & & 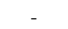 & & - & & - \\
\hline & & $+1.2 \%$ & & & & $-0.2 \%$ & & & & $+0.1 \%$ & & $-0.1 \%$ \\
\hline & & $-1.4 \%$ & & & & -0. & & & & $+1.1 \%$ & & $+1.5 \%$ \\
\hline & & -1 & & & & +1 & & & 0.5013 & $+1.5 \%$ & 0.5668 & $+1.0 \%$ \\
\hline Pre-Pair BERT & 0.4515 & $-0.4 \%$ & 0.5238 & $-0.6 \%$ & 0.4752 & $+1.2 \%$ & 0.5285 & $+0.7 \%$ & 0.4979 & $+0.9 \%$ & 0.5594 & $-0.3 \%$ \\
\hline
\end{tabular}

\section{A Results using the BERT core with all marking strategies}

\section{A.1 Zero-shot transfer setting}

Table 18 shows the full results obtained using all the proposed strategies on Robust04 and GOV2 collections at cutoff 100 and 1,000. We report results using the title, description and hybrid runs. The results at cutoff 1,000 complement the reported results in Tables 6 and 8 For the 100-cutoff results, they complement the results of Table 10 for the zero-shot transfer section. We report the results at cutoff 100 for direct comparison with the multi-phase finetuning setting where we only rerank the top- 100 documents retrieved by BM 25 as a trade-off between effectiveness and efficiency.

\section{A.2 Multi-phase fine-tuning setting}

Table 19 shows the results obtained using the multi-phase fine-tuning setting described in section 5.3 for all our models using all proposed marking strategies. These results expand those presented in Table 10 
Table 19: Reranking effectiveness in the multi-phase fine-tuning setting of the different models on Robust04 and GOV2 collections. Best results are highlighted in bold. Significant improvements over the Vanilla baseline with $p<0.05$ and $p<0.01$ are indicated with $\dagger$ and $\ddagger$ respectively. Significant inferiority with $p<0.05$ is marked with $*$.

\begin{tabular}{|c|c|c|c|c|c|c|c|c|c|c|c|c|}
\hline Robust04 & \multicolumn{4}{|c|}{ Title run } & \multicolumn{4}{|c|}{ Description run } & \multicolumn{4}{|c|}{ Hybrid run } \\
\hline Model & $\mathrm{ADCG} @ 20$ & & $\mathrm{P} @ 20$ & & nDCG@2 & & $\mathrm{P} @ 20$ & & nDCG@21 & & $\mathrm{P} @ 20$ & \\
\hline BM25 & 0.4240 & - & 0.3631 & - & 0.4058 & - & 0.3345 & - & 0.4240 & - & 0.3631 & - \\
\hline BM25+RM3 & 4407 & - & & - & & - & 0.3661 & - & 0.4407 & - & & - \\
\hline Vanilla BERT & 4995 & - & 0.4275 & - & 5368 & - & 4492 & - & .5546 & - & .4715 & - \\
\hline Sim-Dor & 4976 & $-0.4 \%$ & 0.4273 & $-0.0 \%$ & 5378 & $+0.2 \%$ & & $-0.5 \%$ & 0.5632 & $+1.6 \%$ & 0.4783 & $+1.4 \%$ \\
\hline Sim-1 & & $+1.3 \%$ & & +2.2 & & $+2.1 \%$ & & $+1.8 \%$ & & $+2.8 \%$ & $0.4815^{\ddagger}$ & $+2.1 \%$ \\
\hline Pre-1 & 5039 & $+0.9 \%$ & 0.4331 & +1 . & 0.5462 & $+1.8 \%$ & 0.4568 & $+1.7 \%$ & & $+1.1 \%$ & 0.4757 & $+0.9 \%$ \\
\hline Pre-Pair & 5021 & $+0.5 \%$ & 0.4333 & $+1.4 \%$ & $0.5532^{\ddagger}$ & $+3.1 \%$ & $0.4631^{\ddagger}$ & $+3.1 \%$ & & $+2.8 \%$ & $0.4821^{\ddagger}$ & $+2.2 \%$ \\
\hline GOV2 & \multicolumn{4}{|c|}{ Title run } & \multicolumn{4}{|c|}{ Description run } & \multicolumn{4}{|c|}{ Hybrid run } \\
\hline Model & nDCG@2 & & $\mathrm{P} @ 20$ & & nDCG@2 & & $P @ 20$ & & nDCG@2 & & $P @ 20$ & \\
\hline BM25 & 4774 & - & 0.5362 & - & 0.4264 & - & & & 0.4774 & 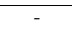 & 0.5362 & - \\
\hline BM25+RM3 & & - & & - & & - & & - & & - & & - \\
\hline Vanilla BERT & 6476 & - & & - & 0.51 & - & & - & 0.590 & - & 0.6604 & - \\
\hline Sim-1 & 5413 & $-1.2 \%$ & 0.62 & -0.9 & 0.51 & $-0.5 \%$ & 0.57 & +0.5 & 0.57 & $-2.6 \%$ & 0.6513 & $-1.4 \%$ \\
\hline & $5743^{\ddagger}$ & $+4.9 \%$ & $0.6540^{\ddagger}$ & +3.8 & $0.5406^{\ddagger}$ & +4.5 & $0.6084^{\ddagger}$ & +5 & & $+1.5 \%$ & 0.6758 & $+2.3 \%$ \\
\hline & & $+2.9 \%$ & & & & & & +5 & & $+1.6 \%$ & & $+1.7 \%$ \\
\hline Pre-Pair BERT & $0.5705^{\ddagger}$ & $+4.2 \%$ & $0.6513^{\ddagger}$ & $+3.3 \%$ & $0.5387^{\ddagger}$ & $+4.1 \%$ & $0.6034^{\ddagger}$ & $+4.5 \%$ & 0.5966 & $+1.0 \%$ & 0.6708 & $+1.6 \%$ \\
\hline
\end{tabular}

\section{B Results using the ELECTRA core with all marking strategies}

\section{B.1 Zero-shot transfer setting}

Table 20 resumes the results of applying all proposed marking strategies on the ELECTRA core model for Robust04 and GOV2 collections. This table complements the results presented in Table 12. We add the results at the reranking cutoff 100 in order to give an idea about the zero-shot setting results without in-domain fine-tuning directly comparable with the multi-phase fine-tuning setting that uses the same reranking threshold of 100 in Table 21

B.2 Multi-phase fine-tuning setting

Table 21 results complements the results presented in Table 13 obtained in the multi-phase fine-tuning setting using all exact match marking strategies proposed in this paper. 
Table 20: Reranking effectiveness in the zero-shot transfer setting of the different models on Robust04 and GOV2 collections. Best results, for each cutoff, are highlighted in bold. Significant improvements over the Vanilla baseline with $p<0.05$ and $p<0.01$ are indicated with $\dagger$ and $\ddagger$ respectively, for the same cutoff. Significant inferiority with $p<0.05$ is marked with $*$. For each measure, the improvement rate over the Vanilla baseline is given (\%).

\begin{tabular}{|c|c|c|c|c|c|c|c|c|c|c|c|c|}
\hline \multirow{3}{*}{$\begin{array}{l}\text { Robust04 } \\
\text { Model } \\
\text { BM25 }\end{array}$} & \multicolumn{4}{|c|}{ Title run } & \multicolumn{4}{|c|}{ Description run } & \multicolumn{4}{|c|}{ Hybrid run } \\
\hline & \multicolumn{2}{|c|}{ nDCG@20 } & \multicolumn{2}{|l|}{$\mathrm{P} @ 20$} & \multicolumn{2}{|c|}{ nDCG@20 } & \multicolumn{2}{|l|}{$\mathrm{P} @ 20$} & \multicolumn{2}{|c|}{ nDCG@20 } & \multicolumn{2}{|l|}{$\mathrm{P} @ 20$} \\
\hline & 0.4240 & - & 0.3631 & - & 0.4058 & - & 0.3345 & - & 0.4240 & - & 0.3631 & - \\
\hline BM25+RM3 & 0.4407 & - & 0.3821 & - & 0.4255 & - & 0.3661 & - & 0.4407 & - & 0.3821 & - \\
\hline \multicolumn{13}{|l|}{ Top-100 } \\
\hline Vanilla ELECTRA & 4712 & & 0.4108 & & 0.4721 & & 0.3988 & - & 0.5103 & - & 0.4323 & \\
\hline Sim-Doc ELF & 0.4680 & $-0.7 \%$ & 0.4054 & $-1.3 \%$ & $0.4804^{\dagger}$ & $+1.8 \%$ & 0.4040 & $+1.3 \%$ & $0.5231^{\ddagger}$ & $+2.5 \%$ & $0.4422^{\dagger}$ & $+2.3 \%$ \\
\hline Sim-Pair & $4820^{\dagger}$ & $+2.3 \%$ & 0.4181 & $+1.8 \%$ & 0.4749 & $+0.6 \%$ & 0.3964 & $-0.6 \%$ & $0.5235^{\dagger}$ & $+2.6 \%$ & $0.4418^{\dagger}$ & $+2.2 \%$ \\
\hline Pre-Doc ${ }_{\mathrm{El}}$ & 4663 & $-1.0 \%$ & 0.4080 & $-0.7 \%$ & 0.4789 & $+1.4 \%$ & 0.4016 & $+0.7 \%$ & 0.5182 & $+1.5 \%$ & 0.4378 & $+1.3 \%$ \\
\hline Pre-Pair ${ }_{1}$ & 0.4668 & $-0.9 \%$ & 0.4064 & $-1.1 \%$ & 0.4740 & $+0.4 \%$ & 0.4002 & $+0.4 \%$ & 0.5169 & $+1.3 \%$ & 0.4416 & $+2.2 \%$ \\
\hline \multicolumn{13}{|l|}{ Top-1000 } \\
\hline Vanilla ${ }_{\mathrm{F}}$ & 416 & - & 0.3833 & - & .4482 & & 0.3831 & - & 0.4782 & & 0.4141 & \\
\hline Sim-Doc ${ }_{E}$ & 4479 & $+1.4 \%$ & 0.3878 & $+1.2 \%$ & $0.4640^{\dagger}$ & $+3.5 \%$ & $0.3948^{\dagger}$ & $+3.1 \%$ & $0.4970^{\ddagger}$ & $+3.9 \%$ & 0.4247 & $+2.6 \%$ \\
\hline Sim-P & $0.4717^{\ddagger}$ & $+6.8 \%$ & $0.4124^{\ddagger}$ & $+7.6 \%$ & 0.4597 & $+2.6 \%$ & 0.3886 & $+1.4 \%$ & $0.5043^{\ddagger}$ & $+5.5 \%$ & 0.4263 & $+2.9 \%$ \\
\hline Pre-Doc ${ }_{F}$ & 4500 & $+1.9 \%$ & 0.3912 & $+2.1 \%$ & $0.4662^{\dagger}$ & $+4.0 \%$ & $0.3948^{\dagger}$ & $+3.1 \%$ & $0.4996^{\ddagger}$ & $+4.5 \%$ & 0.4251 & $+2.7 \%$ \\
\hline Pre-Pair electra & 4511 & $+2.2 \%$ & 0.3934 & $+2.6 \%$ & 0.4537 & $+1.2 \%$ & 0.3878 & $+1.2 \%$ & $0.4936^{\dagger}$ & $+3.2 \%$ & 0.4245 & $+2.5 \%$ \\
\hline GOV2 & \multicolumn{4}{|c|}{ Title run } & \multicolumn{4}{|c|}{ Description run } & \multicolumn{4}{|c|}{ Hybrid run } \\
\hline Model & nDCG@2 & & $P @ 20$ & & nDCG@2 & & $P @ 20$ & & nDCG@2 & & $P @ 20$ & \\
\hline BM25 & 0.4774 & - & 0.5362 & - & 0.4264 & - & 0.4705 & - & 0.4774 & - & 0.5362 & - \\
\hline BM25+RM3 & 851 & - & 0.5634 & - & & - & 0.4966 & - & 0.4851 & - & 0.5634 & - \\
\hline \multicolumn{13}{|l|}{ Top-100 } \\
\hline Vanilla ELECTR & 5278 & - & 0.6094 & - & 0.5153 & - & 0.5785 & - & 0.5803 & - & 0.6617 & - \\
\hline & 342 & $+1.2 \%$ & 0.6188 & $+1.5 \%$ & 0.51 & $-0.6 \%$ & 0.5795 & $+0.2 \%$ & 0.5761 & $-0.7 \%$ & 0.6527 & $-1.4 \%$ \\
\hline & 5387 & $+2.1 \%$ & 0.6171 & $+1.3 \%$ & 0.52 & $+1.0 \%$ & & $+1.3 \%$ & 0.5801 & $-0.0 \%$ & 0.6587 & $-0.5 \%$ \\
\hline & & $+1.4 \%$ & 0.61 & & & -1.3 & 0.5 & -1 & 0.5779 & $-0.4 \%$ & 0.6557 & $-0.9 \%$ \\
\hline Pre-Pair ELECTRA & 0.5306 & $+0.5 \%$ & 0.6131 & $+0.6 \%$ & 0.5108 & $-0.9 \%$ & 0.5775 & $-0.2 \%$ & 0.5760 & $-0.7 \%$ & 0.6557 & $-0.9 \%$ \\
\hline \multicolumn{13}{|l|}{ Top-1000 } \\
\hline Vanilla El & 668 & - & 0.5332 & - & & - & 0.5601 & - & .5147 & - & & - \\
\hline Sim-Doc & 796 & $+2.7 \%$ & $0.5530^{\dagger}$ & $+3.7 \%$ & 0.4958 & $-0.6 \%$ & 0.5544 & $-1.0 \%$ & 0.5198 & $+1.0 \%$ & 0.5930 & $+2.9 \%$ \\
\hline Sim-Pair & $0.4881^{\dagger}$ & $+4.6 \%$ & $0.5577^{\ddagger}$ & $+4.6 \%$ & 0.5030 & $+0.9 \%$ & 0.5634 & $+0.6 \%$ & 0.5249 & $+2.0 \%$ & 0.5923 & $+2.7 \%$ \\
\hline Pre-Doc ${ }_{E}$ & $0.4845^{\dagger}$ & $+3.8 \%$ & $0.5530^{\ddagger}$ & $+3.7 \%$ & 0.4981 & $-0.1 \%$ & 0.5560 & $-0.7 \%$ & 0.5212 & $+1.3 \%$ & 0.5883 & $+2.0 \%$ \\
\hline Pre-Pair electra & 0.4820 & $+3.3 \%$ & $0.5513^{\dagger}$ & $+3.4 \%$ & $0.4828^{*}$ & $-3.2 \%$ & 0.5419 & $-3.2 \%$ & 0.5075 & $-1.4 \%$ & 0.5711 & $-0.9 \%$ \\
\hline
\end{tabular}

Table 21: Reranking effectiveness in the multi-phase fine-tuning setting of the different models on Robust04 and GOV2 collections. Best results are highlighted in bold. Significant improvements over the Vanilla baseline with $p<0.05$ and $p<0.01$ are indicated with $\dagger$ and $\ddagger$ respectively. Significant inferiority with $p<0.05$ is marked with $*$.

\begin{tabular}{|c|c|c|c|c|c|c|c|c|c|c|c|c|}
\hline Robust04 & \multicolumn{4}{|c|}{ Title run } & \multicolumn{4}{|c|}{ Description run } & \multicolumn{4}{|c|}{ Hybrid run } \\
\hline Model & nDCG@2 & & $P @ 20$ & & nDCG@2 & & $\mathrm{P} @ 20$ & & nDCG@2 & 0 & $P @ 20$ & \\
\hline BM25 & 0.4240 & - & 0.3631 & & 0.4058 & - & 0.3345 & - & 0.4240 & - & 0.3631 & - \\
\hline BM25+RM3 & 0.4407 & - & 0.3821 & - & 0.4255 & - & 0.3661 & - & 0.4407 & - & 0.3821 & - \\
\hline Vanilla ELECTRA & 0.5375 & - & 0.4560 & & 0.5676 & - & 0.4663 & - & 0.5901 & - & 0.4902 & \\
\hline Sim-Doc ELECTRA & 0.5367 & $-0.1 \%$ & 0.4560 & $+0.0 \%$ & 0.5662 & $-0.2 \%$ & 0.4683 & $+0.4 \%$ & 0.5893 & $-0.1 \%$ & 0.4912 & $+0.2 \%$ \\
\hline Sim-Pair ELECTRA & 0.5380 & $+0.1 \%$ & 0.4564 & $+0.1 \%$ & 0.5686 & $+0.2 \%$ & 0.4705 & $+0.9 \%$ & 0.5927 & $+0.4 \%$ & 0.4942 & $+0.8 \%$ \\
\hline Pre-Doc ELECTRA & 0.5338 & $-0.7 \%$ & 0.4590 & $+0.7 \%$ & 0.5705 & $+0.5 \%$ & 0.4697 & $+0.7 \%$ & 0.5889 & $-0.2 \%$ & 0.4926 & $+0.5 \%$ \\
\hline Pre-Pair ELECTRA & 0.5390 & $+0.3 \%$ & 0.4566 & $+0.1 \%$ & 0.5677 & $+0.0 \%$ & 0.4699 & $+0.8 \%$ & 0.5930 & $+0.5 \%$ & 0.4970 & $\begin{array}{r}+1.4 \% \\
\end{array}$ \\
\hline GOV2 & \multicolumn{4}{|c|}{ Title run } & \multicolumn{4}{|c|}{ Description run } & \multicolumn{4}{|c|}{ Hybrid run } \\
\hline Model & nDCG@2 & & $P @ 20$ & & nDCG@2 & & $P @ 20$ & & nDCG@2 & & $P @ 20$ & \\
\hline BM25 & 0.4774 & - & 0.5362 & - & 0.4264 & - & 0.4705 & - & 0.4774 & - & 0.5362 & - \\
\hline BM25+RM3 & 0.4851 & - & 0.5634 & - & 0.4212 & - & 0.4966 & - & 0.4851 & - & 0.5634 & - \\
\hline Vanilla ELECTRA & 0.5784 & - & 0.6621 & & 0.5629 & - & 0.6279 & - & 0.6149 & - & 0.6862 & - \\
\hline Sim-Doc ELECTRA & 0.5891 & $+1.8 \%$ & 0.6685 & $+1.0 \%$ & $0.5044^{*}$ & $-10.4 \%$ & $0.5758^{*}$ & $-8.3 \%$ & 0.6120 & $-0.5 \%$ & 0.6926 & $+0.9 \%$ \\
\hline Sim-Pair ELECTRA & 0.5868 & $+1.5 \%$ & 0.6661 & $+0.6 \%$ & 0.5552 & $-1.4 \%$ & 0.6225 & $-0.9 \%$ & 0.6133 & $-0.3 \%$ & 0.6926 & $+0.9 \%$ \\
\hline Pre-Doc ELECTRA & 0.5841 & $+1.0 \%$ & 0.6634 & $+0.2 \%$ & 0.5524 & $-1.9 \%$ & 0.6188 & $-1.4 \%$ & 0.6130 & $-0.3 \%$ & 0.6852 & $-0.1 \%$ \\
\hline Pre-Pair ELECTRA & $0.5920 \dagger$ & $+2.4 \%$ & 0.6718 & $+1.5 \%$ & $0.5486^{*}$ & $-2.5 \%$ & $0.6134^{*}$ & $-2.3 \%$ & 0.6207 & $+0.9 \%$ & 0.6956 & $+1.4 \%$ \\
\hline
\end{tabular}

